STONE CENTER ON SOCIO-ECONOMIC INEQUALITY

WORKING PAPER SERIES

No. 04

The Long Run Evolution of Absolute Intergenerational Mobility

Yonatan Berman

February 2020

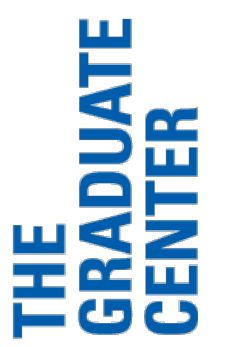




\title{
The Long Run Evolution of Absolute Intergenerational Mobility
}

\author{
Yonatan Berman*†
}

\begin{abstract}
This paper combines cross-sectional and longitudinal income data to present the evolution of absolute intergenerational income mobility in ten developed economies in the 20th century. Absolute mobility decreased during the second half of the 20th century in all these countries. Increasing income inequality and decreasing growth rates have contributed to the decrease. Yet, growth is the dominant contributor in most countries. We show that detailed panel data are unnecessary for estimating absolute mobility over the long run.
\end{abstract}

Keywords: Mobility, inequality, copula modeling

JEL Codes: D3, E2, H0, J6

*London Mathematical Laboratory, The Graduate Center and Stone Center on Socio-Economic Inequality, City University of New York, y.berman@lml.org.uk

${ }^{\dagger}$ I wish to thank Alex Adamou, Jack Blundell, François Bourguignon, Raj Chetty, Paul Goldsmith-Pinkham, Markus Jäntti, Yoash Shapira, Tim Smeeding, and Daniel Waldenström, as well as numerous seminar and conference participants for helpful discussions and comments. 


\section{Introduction}

The question whether next generations will be better off than previous ones is central in the recent public and economic debate. Chetty et al. (2014므) discuss a "growing public perception that intergenerational income mobility $[\ldots]$ is declining in the United States." Others argue that "people's frustrations [... are] rooted in the fear that their kids won't be better off than they were." (Obama, 2013) This can be quantified as absolute intergenerational mobility - the fraction of children with higher real incomes than their parents at the same age. This measure captures the chances of children to have a higher standard of living than their parents.

Chetty et al. (2017) studied the historical evolution of absolute mobility in the United States. They showed that it fell from around 90\% for children born in 1940 to $50 \%$ for children born in 1980 . They found that two-thirds of that decline are due to changes in the income distribution. A third of the decline is due to slower growth rates over the past thirty years relative to the 1940s and 1950s. Yet, there is little evidence on the experience in other countries and more birth cohorts. Thus, it is crucial to test how general these findings are in terms of absolute mobility levels, historical trends, and sources.

Estimating absolute intergenerational mobility for longer time periods and additional countries poses challenges. First, matching parents and children requires historical panel data. These are usually rare, do not cover the entire income distribution, or are available for a limited range of birth cohorts. Second, reliable income data are many times disjoint from microdata such as age and gender. This complicates the identification of parents and children. These issues are of particular importance when considering early 20th century, or earlier, cohorts. In such cases, the existing data sources on income are limited.

This paper presents the long run evolution of absolute intergenerational mobility in income in a large group of developed economies. In France, Sweden and the United States it was possible to estimate absolute mobility for birth cohorts of the early 20th century. This goes well beyond the existing literature on absolute mobility.

Our approach combines the marginal income distributions for the parent and child generations and their copula - the joint distribution of parent and child income ranks. It overcomes the described challenges. We find that historical panel data are unnecessary for estimating absolute mobility. Estimates of absolute mobility depend mainly on the marginal income distributions, and their copula plays a minor role in determining absolute mobility. We also find that changes in the copula cannot explain the long run evolution of absolute mobility, and only changes in the marginal distributions can. In short, we provide robust evidence that absolute mobility is driven primarily by economic growth, secondarily by changes in inequality, and thirdly (and weakly) by relative mobility. ${ }^{1}$

\footnotetext{
${ }^{1}$ Intergenerational mobility is typically divided into two classes: relative and absolute. Relative measures gauge children's propensity to occupy a different position in the income distribution than their parents. The copula fully encodes relative mobility. Absolute measures gauge their propensity to have higher incomes than their parents in
} 
These observations make the estimation of absolute mobility possible even for countries in which panel data are scarce. We show that very limited data are enough for capturing the long run evolution of absolute mobility. We then combine available data on intergenerational copulas and historical income distributions to provide absolute mobility estimates in developed countries over the course of the 20th century.

We find a large and nearly monotonic decrease in the chances of children to have a higher income than their parents in all studied countries during the post-war period, from $80 \%-100 \%$ to $50 \%-70 \%$. This decrease followed a rapid increase in absolute mobility from the early 1900s until World War II, reflecting the economic boom and the decreasing income inequality during the three decades that followed the end of World War II. This paper thus extends the findings of Chetty et al. (2017) for the United States. It shows that levels of absolute mobility were high even before World War II. They peaked around the 1940 birth cohort.

The factors driving the decrease in absolute mobility differ among countries. In Australia and the United States the decrease is mainly due to increasing income inequality. Yet, in other countries, notably Denmark, France and Japan, the slow economic growth of the past several decades is the key contributor to a similar decrease. Despite higher growth rates and regardless of the "American Dream" ethos, the absolute intergenerational mobility in the United States for late 1970s and early 1980s birth cohorts is among the lowest within the group of countries we studied.

To inform and interpret the empirical findings, we study a simple model. The model provides a relationship between absolute mobility, income growth, income inequality and relative mobility. It describes well the long run evolution of absolute mobility, even when only limited information on income distributions is taken into account. For example, the knowledge of historical income growth rates and Gini coefficients is satisfactory for reliable absolute mobility estimation. We also show that in this model, ceteris paribus, absolute and relative mobility are inversely related. In practice, despite this relationship, countries characterized by high relative mobility, such as the Nordic countries, are also characterized by high absolute mobility. As described, the effects of growth and inequality on absolute mobility are more important than that of relative mobility.

Relative mobility has been studied for decades. ${ }^{2}$ Yet, investigations of absolute mobility in income remain "scarce, mainly because of the lack of large, high-quality panel data sets linking children to their parents." (Chetty et al., 2017) Absolute mobility has been studied so far mainly in the context of class, education, and occupation, which are central in the sociological mobility literature. ${ }^{3}$ The differences in outcome variables and the different nature of measures used make the results in this literature difficult to compare to this paper. Yet, we note that Lipset and Rogoff (1954) had already identified that "the overall pattern of social mobility appears to be much the same in the industrial

real terms.

${ }^{2}$ See, for example, Becker and Tomes (1979); Borjas (1992); Mazumder (2005); Aaronson and Mazumder (2008); Lee and Solon (2009); Corak (2013); Chetty et al. (2014b); Berman (2019).

${ }^{3}$ See, for example, Lipset and Rogoff (1954); Lipset and Zetterberg (1959); Erikson, Goldthorpe and Portocarero (1979); Goldthorpe (1987); Erikson and Goldthorpe (1992); Breen and Jonsson (2005); Breen and Rottman (2014). 
societies of various Western countries."

Our contribution is thus threefold. First, from an empirical perspective, the primary contribution of this paper is to provide new series on absolute intergenerational mobility in a large group of developed countries. In particular, we provide evidence on absolute intergenerational mobility for the early 20th century birth cohorts. We find that in all studied countries absolute mobility decreased during the second half of the 20th century.

We also describe the effect of income growth and income inequality on absolute mobility and decompose the changes in absolute mobility into the contribution of each. We show that the decrease of income growth rates is the main determinant of changes in absolute mobility in most countries. In Australia and the United States, and to a much lesser extent in Finland and the United Kingdom, increasing inequality was more important for the long run trend of absolute mobility than growth.

Second, from a methodological perspective, we describe in detail the low sensitivity of absolute intergenerational mobility estimates to data limitations. This allows estimating absolute mobility in the long run without the necessity for detailed panel data. Notably, we show that copulas of different countries share a similar form. Together with the low sensitivity of absolute mobility to changes in the copula, this enables characterizing copulas with a single parameter, such as the rank correlation, when estimating absolute mobility. The same methodology could be applied to other countries in the years ahead. Also, since household surveys are a common practice in most countries today, it will be possible to continue tracking absolute mobility even without detailed panel data, which are still much less common.

Third, from a theoretical perspective, we derive a mathematical relationship between absolute mobility, income growth, income inequality changes and relative mobility. It proves as a powerful way for estimating absolute mobility with very limited data. Namely, total income growth between generations and the Gini coefficients are enough for reliable absolute mobility estimation. This relationship also allows studying properties of absolute mobility previously uncovered.

The paper is organized as follows. Section 2 lays out our methodology, addressing the necessity of panel data for producing reliable estimates of absolute mobility. Section 3 specifies our data sources and presents the main results. Section 4 discusses a simplified model for the relationship between absolute mobility, income growth, income inequality and relative intergenerational mobility. We conclude in Section 5.

\section{Methodology}

In an ideal setting, measuring the rate of absolute intergenerational mobility is trivial. For every birth cohort of children we would trace back their parents and compare their individual or household incomes at a certain age. However, such data are usually available for small samples, do not cover 
the whole income distribution, or available for a very limited range of birth cohorts. In many countries, such data are rare and may suffer from large measurement errors (Solon, 1992; Chetty et al., 2014a). Using such data for estimating typical measures of relative income mobility over a long period of time may be challenging and unreliable.

Yet, in the case of absolute intergenerational mobility, one is able to provide reliable estimates, even in the absence of historical detailed panel data. Our methodology builds on the approach of Chetty et al. (2017). We use repeated cross-sections and combine them using a copula, the joint distribution of parent and child income ranks. It follows that the rate of absolute mobility $A$ is (see equation (2) in Chetty et al. (2017)):

$$
A=\int \mathbf{1}_{\left\{Q^{c}\left(r^{c}\right) \geq Q^{p}\left(r^{p}\right)\right\}} C\left(r^{c}, r^{p}\right) d r^{c} d r^{p},
$$

where $r^{c}$ and $r^{p}$ are the child and parent income ranks, respectively; $Q^{c}$ and $Q^{p}$ are the respective quantile functions; $C$ is the copula.

Using copulas estimated for different countries and birth cohorts we identify two crucial points.

First, the basic structure of realistic copulas is similar - when two realistic copulas differ in a measure of relative mobility, they differ proportionally in other relative mobility measures. This observation implies that using a single measure of relative mobility provides a good approximation for intergenerational copulas. In order to validate this point we use 28 different copulas measured for different birth cohorts, different countries, and for pre- and post-tax incomes. We then compare them in terms of different measures of relative mobility. Each copula is considered as a bi-stochastic matrix $P \in \mathscr{P}(N)$, where $p_{i j}$ represents the probability of transferring to quantile $j$ (child) for those starting in quantile $i$ (parent) and $N$ is the number of income quantiles.

We consider four standard measures of relative mobility, which are defined on $P$ : Spearman's rank correlation (Spearman, 1904); Bartholomew's index (Bartholomew, 1967); Average absolute nonzero jump; Shorrocks' trace index (Shorrocks, 1978). The different measures are mathematically related, however they are not linearly dependent. Specifically, it is possible to construct matrices which have the same trace index, but very different rank correlation, average absolute non-zero jump measure or Bartholomew's index and vice versa (Bartholomew, 1967; Shorrocks, 1978; Atkinson and Bourguignon, 1982; Atkinson, 1983). In practice, we find that the correlation between each two measures across the 28 copulas is between 0.93 and 1 . In other words, despite the a priori independence of the relative mobility measures, the different relative mobility measures are, in fact, almost linearly related across time and countries, for pre- and post-tax incomes. Fig. 1 presents the comparison of the different relative mobility measures (for the exact definitions of the measures used see Appendix B).

We conclude, therefore, that the shape of the copulas is similar and they can be practically summarized by a single parameter. We use the rank correlation since in many countries relative mobility is reported using the rank correlation or the intergenerational elasticity, from which the rank cor- 

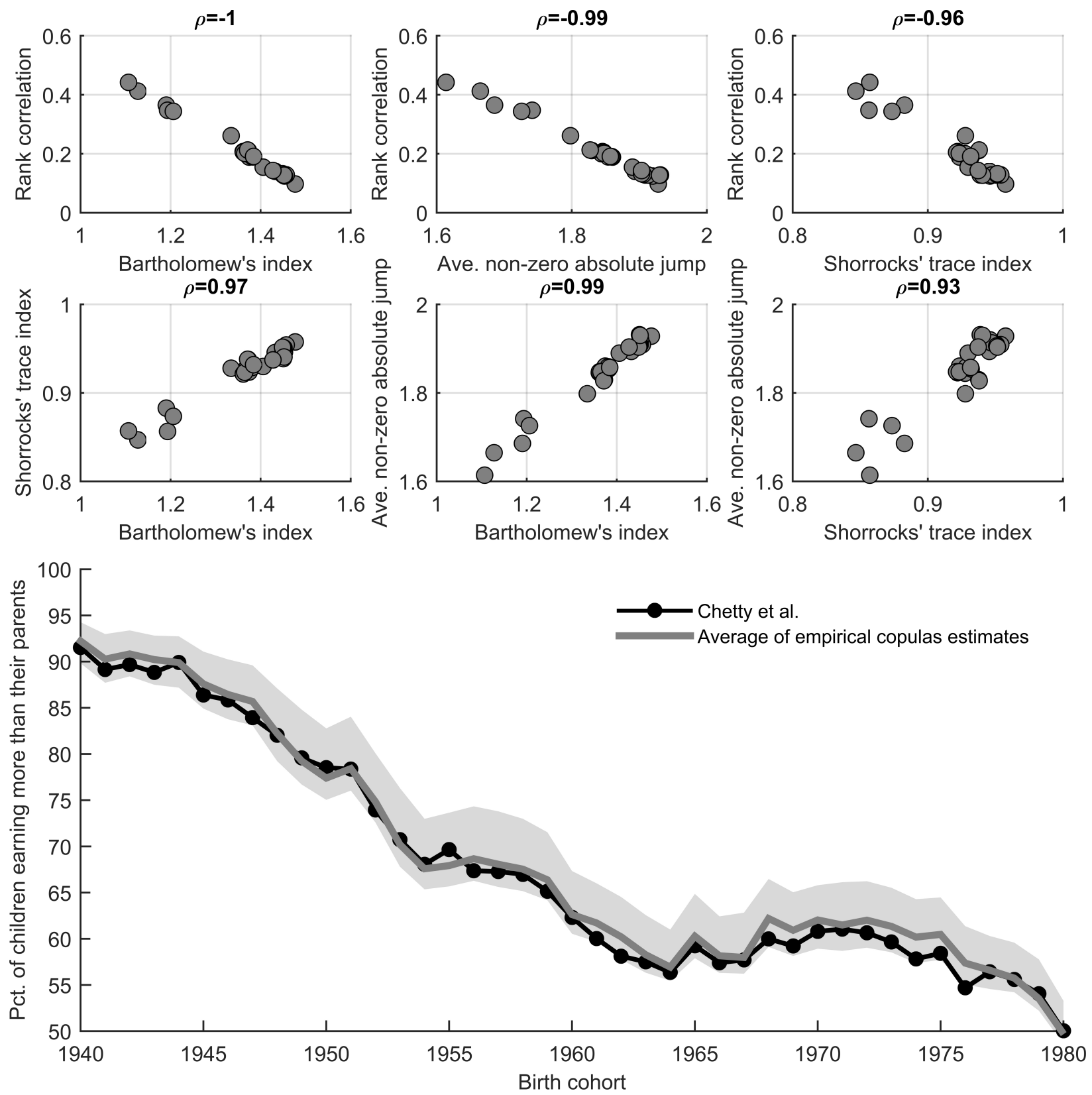

Figure 1: Top) A comparison between relative mobility measures in 28 empirical copulas ( $\rho$ is the Pearson correlation between each two relative mobility measures. The standard error on the correlation values was lower than 0.01 in all cases.); Bottom) The effect of changes in copula on absolute intergenerational mobility in the United States. Absolute mobility was estimated using the marginal distributions used in Chetty et al. (2017) and 28 empirical copulas for Denmark, Finland, Germany, Norway, Sweden, the United Kingdom and the United States. The shaded gray area is the area covered by the various absolute mobility estimates. The gray curve is the arithmetic mean of all 28 estimates in each year. The black circles are the estimates reported in Chetty et al. (2017).

relation can be deduced. The entire copula is only rarely reported. Also, the rank correlation is more empirically robust than other measures (Chetty et al., 2014a). 
The second crucial point is that the sensitivity of the absolute mobility estimates to plausible changes in the copula is low. In particular, plausible changes in relative mobility measures cannot explain long term changes in absolute mobility. Therefore, assuming a fixed copula in time will provide meaningful and reliable estimates of absolute mobility. Together with the observation on the copula structure, it would allow us to argue that the marginal income distributions and a single observation of a relative mobility measure, such as the rank correlation, can provide reliable estimates of absolute mobility for various countries.

Figure 1 demonstrates that estimating the absolute mobility in the United States with different copulas, some of which are very different from the one characterizing the United States, results in a similar evolution in time. The estimates obtained using the various copulas differ from the benchmark estimates of Chetty et al. (2017) by 0.14 percentage points on average. We conclude that letting the copula change in time within the boundaries defined by realistic copulas, cannot explain more than a change of several percentage points in absolute mobility over a long period of time. Thus, only changes in marginal distributions can possibly explain the long run absolute mobility trend observed.

We note that conceptually, the same rank correlation could deliver different absolute mobility estimates. Yet, the similar shape of empirical copulas described above, makes this practically implausible (see Appendix C). We also test the sensitivity of absolute mobility to different standard copula models (see in Appendix D), showing that for the same rank correlation, this sensitivity is very small.

\section{Results}

\subsection{Data}

The previous section demonstrated that absolute intergenerational mobility mainly depends on the income distributions of parents and children, rather than on their copula. Following the steps of Chetty et al. (2017) requires such distributions for 30-year-olds only, in years that are 30 years apart. Namely, for estimating absolute mobility for children born in 1980, we would need to possess the marginal income distribution of 30-year-olds in 1980 and in 2010. In the United States this became possible combining census, tax, and survey data for 1940-1984 birth cohorts. In France this has also become possible for 1970-1984 birth cohorts, using the tax data studied in Garbinti, Goupille-Lebret and Piketty (2018). However, we have good knowledge of the income distribution of the entire adult population in these countries in earlier years, based on tax data, surveys and national accounts.

Such data, in different levels of accuracy and detail, are available from The World Inequality Database (2018). When available, we use pre-tax total income data in the equal-split assumption: individuals in tax units that are composed of more than one income-contributing individuals are 
assumed to contribute each an equal part to the total income. The equal-split assumption is compatible with the income specification in Chetty et al. (2017), using total family income of children and parents rather than individual incomes (see Alvaredo et al. (2016) for more details on the equal-split assumption). When the incomes of equal-split adults are not available we use tax units as the unit of observation and when these are not available we use individual incomes. Appendix E discusses the potential effect the differences in unit of observation may have on the results.

Data for the rank correlation were taken from different studies. ${ }^{4}$ For each country we consider a nominal value, a lower bound - 0.1 (smaller than the smallest value estimated in any of the countries), and an upper limit - 0.5 (larger than the largest value estimated in any of the countries). These bounds not only account for possible measurement errors, but also for the stable rank correlation we use for each country. In practice, the rank correlation may have changed over time. Using these bounds we account for the possible effect of such changes. The rank correlation values used and the cohorts covered by the data are detailed in Appendix F.

\subsection{Absolute Intergenerational Mobility in Developed Economies}

We estimate long run absolute intergenerational mobility series in 10 developed countries from 4 continents: Australia, Canada, Denmark, Finland, France, Japan, Norway, Sweden, the United Kingdom and the United States. We assume that the rank correlation in each country is stable over time and consider a range of values - a nominal value, a lower bound (0.1) and an upper bound (0.5) (see above).

We use the generalized Pareto curve interpolation method (Blanchet, Fournier and Piketty, 2017) to get the marginal income distributions. We use the method to generate large simulated samples of the pre-tax income distribution. For some years the data cover only the top $10 \%$ of the distribution. The generalized Pareto curve interpolation method might not be able to describe well the income distribution within the bottom $90 \%$ in such cases. In the next section we show that such data limitations would have a small impact on the absolute mobility estimates in practice. This leads to a powerful conclusion: In the absence of detailed panel data and with limited information on the income distribution shape, it is possible to estimate the evolution of absolute mobility in the long run.

The analysis overcomes another data limitation. The available historical data include all adult population and it is not possible to restrict the data to 30-year-olds only (or specific marital status, family size, etc.). Yet, this difference would have a small effect in practice if income growth and inequality among 30-year-olds evolve similarly to that of the entire adult population. In the United

\footnotetext{
${ }^{4}$ For the countries in which the intergenerational elasticity was reported, rather than the rank correlation, we use the relationship $\rho=\frac{\sigma_{p}}{\sigma_{c}} \beta$, where $\sigma_{p}$ and $\sigma_{c}$ are the standard deviations of the parent and child marginal log-income distributions and $\beta$ is the estimated intergenerational income elasticity. The rank correlation is approximated by $\rho_{S} \approx \frac{6 \arcsin (\rho / 2)}{\pi}$ (see Trivedi and Zimmer $(2007)$ ).
} 
States, for example, the differences between our estimates of absolute mobility and those of Chetty et al. (2017) are small. They are always less than 2 percentage points, excluding several birth cohorts in the mid 1940s. Two effects drive the small difference - income growth is slightly lower among 30 -year-olds than among the entire adult population, while inequality among 30-year-olds is also lower. The effects of these differences on absolute mobility almost cancel out (see also Section 4). Similarly, in France, "[... inequality] is almost as large within each age group as for the population taken as a whole." (Garbinti, Goupille-Lebret and Piketty, 2018) This cancels out with the lower growth among 30-year-olds compared to the whole population.

The difference may be large in some countries. Thus, we currently restrict our analysis to developed countries only, in which the age-income structure was well-studied. The dependence of growth and inequality on age groups changes only little the picture for the entire adult population in these countries. Therefore, it is possible to use the historical data of the entire adult population to obtain a good understanding of the long run evolution of absolute mobility, rather than a very accurate picture of the absolute mobility for a specific birth cohort. In France, detailed tax data from 1970 onward (Garbinti, Goupille-Lebret and Piketty, 2018) allow testing the robustness of the estimates to this assumption for three birth cohorts (see Appendix G). We also find that the simplifying assumptions made here have only a small effect and our results match the existing empirical evidence for Canada (Ostrovsky, 2017) and the United States (Chetty et al., 2017). They are also in accordance with most of the recent evidence on other countries (Blanden, Machin and Rahman, 2019; Liss, Korpi and Wennberg, 2019; Manduca et al., 2019).

Figure 2 presents the main results. They show that the trend of absolute mobility found in the United States is similar to that in many other developed countries. Absolute mobility decreased in all countries studied for post-World War II birth cohorts. In some countries, especially France, Japan and the United States, the decrease is very dramatic. In these countries absolute mobility decreased from more than $90 \%$ for 1940s birth cohorts to less than $60 \%$ for 1980 s birth cohorts. The Nordic countries, characterized by low income inequality and high relative mobility (Corak, 2013), also stand out in high absolute mobility. We also find that absolute mobility in France, Sweden and the United States increased for the children born in the 1910s-1940s, the direct beneficiaries of the Trente Glorieuses in France and the Rekordåren in Sweden. These findings also extend those of Chetty et al. (2017). They indicate that the levels of absolute mobility were high even before World War II in the United States, and peaked around the 1940 birth cohort.

Figure 2 also shows that the effects of the stable rank correlation assumption and the uncertainty in its value are small. We assumed the rank correlation was within the range $[0.1,0.5]$, covering a wider range of values than the combined range of estimated values in all countries considered. Then we estimated the absolute intergenerational mobility in each country assuming the rank correlation is within this range. These estimates are almost identical to the baseline estimates in all countries. We conclude that the simplifying assumptions may potentially lead to an estimation error of up to several percentage points in absolute mobility. However, as explained, they cannot explain long run 

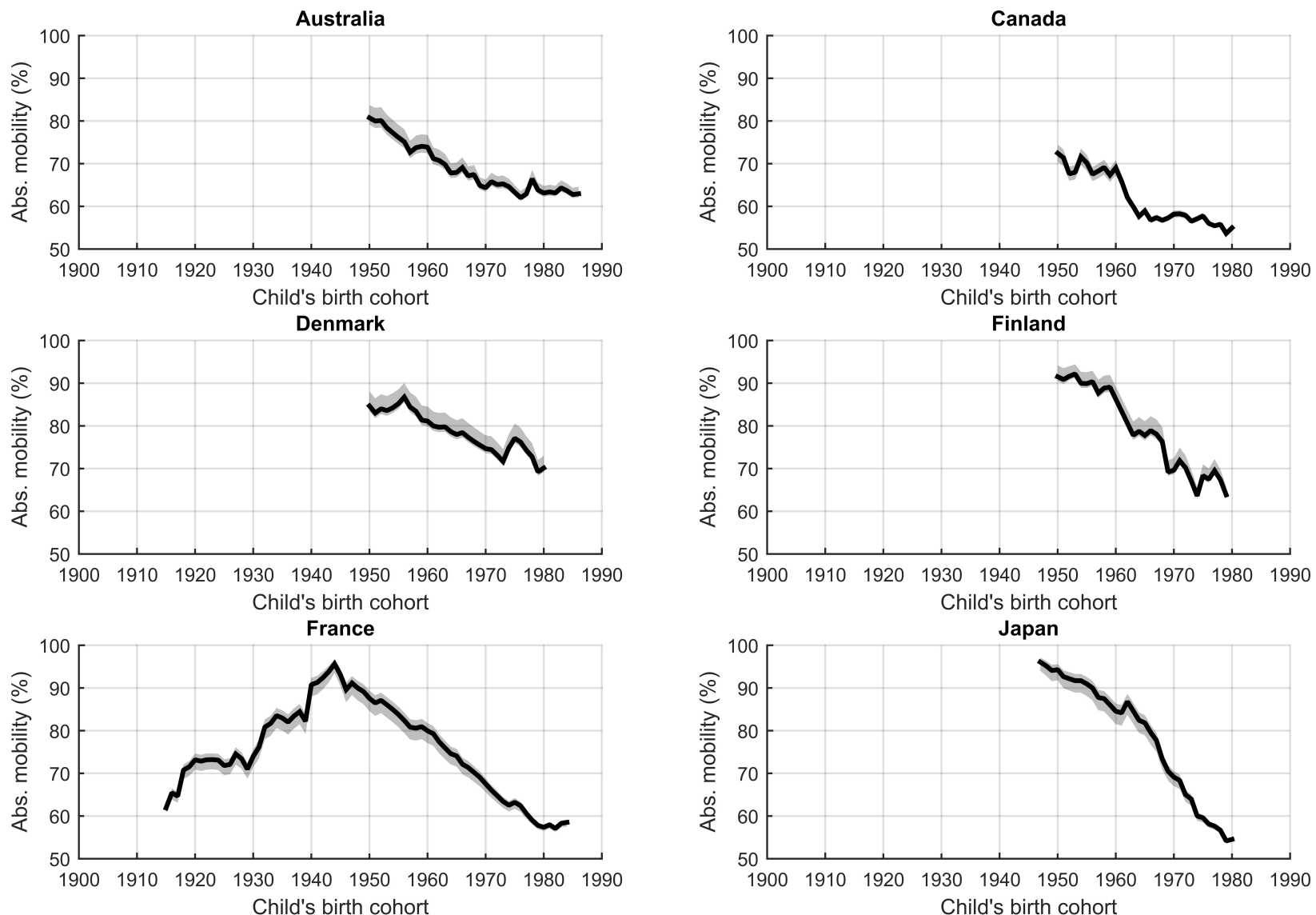

Norway
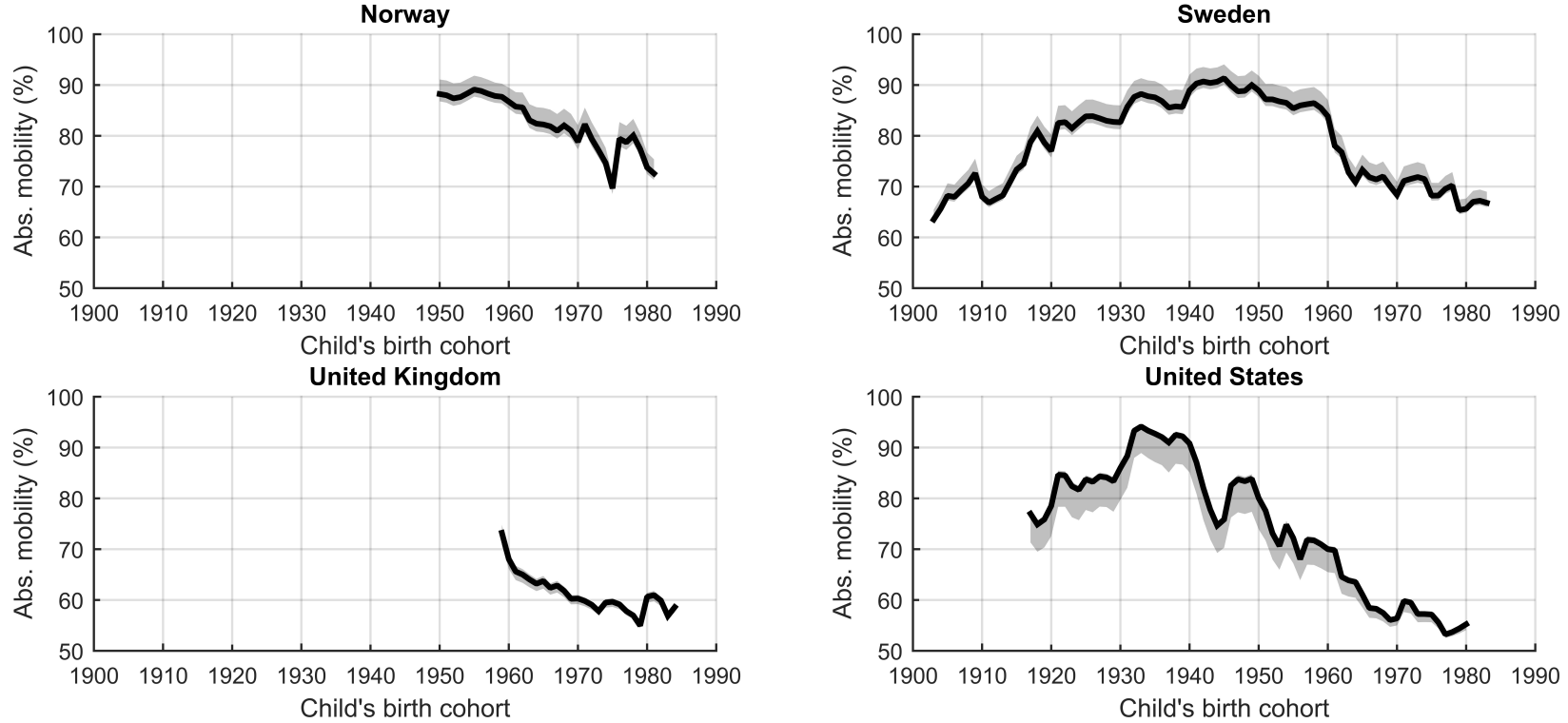

Figure 2: The evolution of absolute intergenerational mobility in developed economies. The absolute mobility based on nominal rank correlations is in black. The shaded gray areas are the ranges covered by the lower and upper bound of the estimates assuming the rank correlation is within the range $[0.1,0.5]$ for each country. In some of the countries the shaded areas are too narrow to be visible. See Appendix $\mathrm{H}$ for the baseline estimates values. 
decreases as significant as we identify. Additional robustness tests are detailed in Appendices E, G and I.

\subsection{Absolute Mobility Decomposition}

Figure 2 shows that in all countries studied absolute mobility decreased during the second half of the 20th century. The size of the decrease differs between country to country. Yet, Fig. 2 cannot answer whether the drivers leading to the decrease are similar or not. Chetty et al. (2017) showed that two-thirds of the decline in absolute mobility in the United States are due to changes in the income distribution. A third of the decline is due to slower growth rates over the past thirty years relative to the 1940 s and 1950 s.

We would now like to address whether the experience in other countries is similar. For that purpose we decompose the absolute mobility trends into contributions of different factors. As noted, we assume that relative mobility is stable over time, so it cannot be the source of long run changes in absolute mobility. Thus, the observed trend is due to two factors - the generally decreasing income growth rates and the generally increasing income inequality.

For each country we estimate the contribution of those factors to the absolute intergenerational mobility trend. For that purpose we produce two counterfactual calculations, in addition to the baseline estimate in each country: one in which the shape of the income distribution is kept constant in time and similar to the earliest distribution in the data (or 1940 in the case of France, Sweden and the United States), but with the average income changing according to its real historical values; and another, in which the distribution shape changes according to historical data, but the annual income growth rate is fixed in time and equal to the average annualized real income growth rate over the entire period considered.

Figure 3 presents these calculations. Countries can be generally divided into two groups - such that the fixed inequality counterfactual scenario follows the baseline estimate (most notably Denmark and France), and such that the baseline estimate is similar to the fixed income growth scenario. In the former case the decrease in absolute mobility is due to the decrease in income growth rates, since fixing inequality has only a small effect. In the latter case, the effect of inequality is more dominant. It is particularly visible for Australia and the United States.

In most of the countries considered, the decreasing income growth rates explain most of the decrease in absolute mobility. Particularly in Canada, France, Japan and the Nordic countries. In Australia, the United Kingdom and the United States the role of increasing income inequality is more important. Despite the similarity in the evolution of absolute mobility in various countries, Australia and the United States are the only countries in which the increasing income inequality played such a dominant role in the decreasing mobility.

We note that this decomposition ignores the interaction between growth and changes in inequality. Yet, evidence for such an interaction are debatable and inconclusive (Bourguignon, 2004; Ostry, 

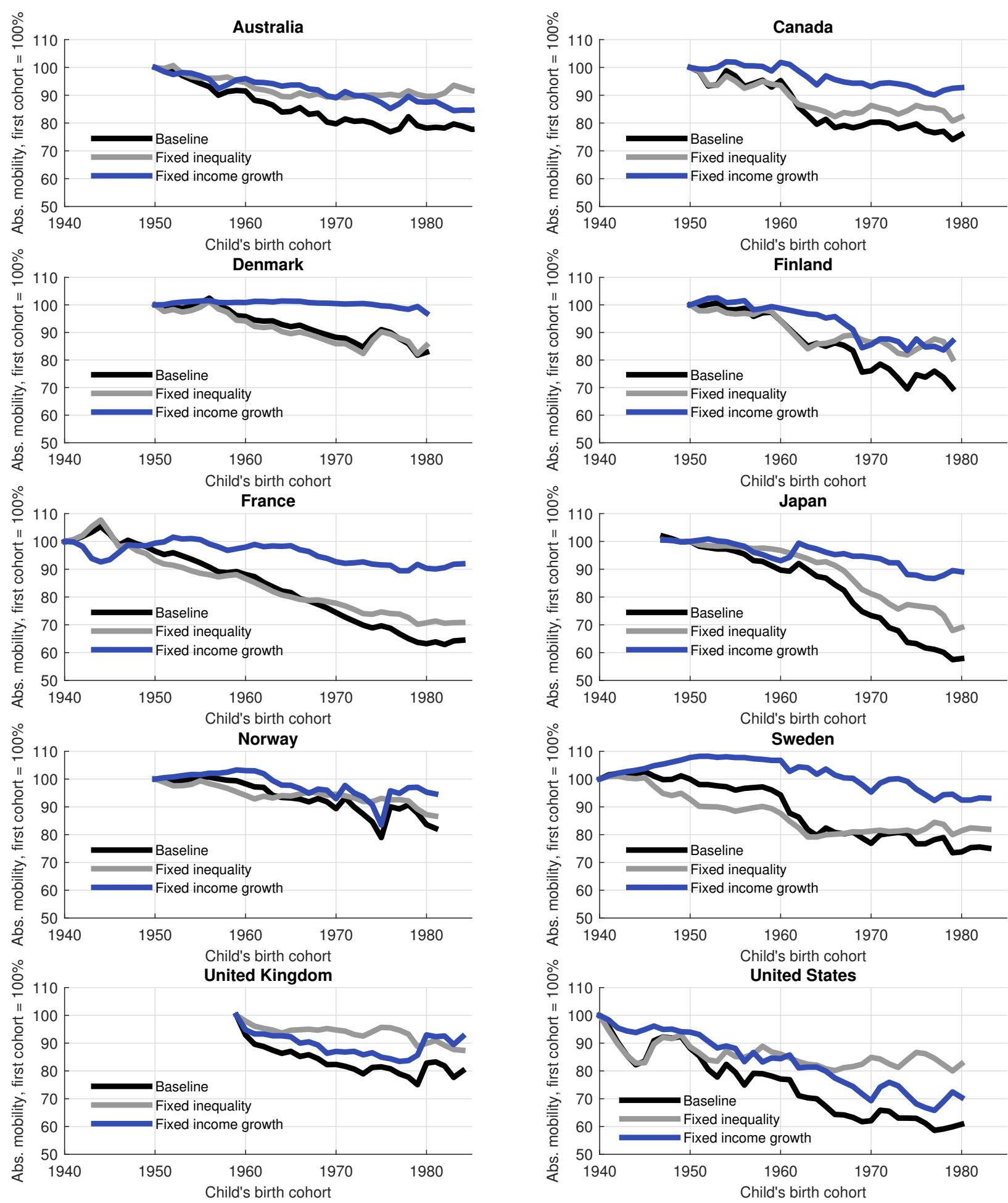

Figure 3: Counterfactual calculations of absolute mobility in a group of developed economies. For comparability, we set the absolute mobility to $100 \%$ in the earliest cohort. See Appendix H for the contribution of each factor to the overall decrease in mobility in all countries. 
Berg and Tsangarides, 2014).

\section{Growth, Inequality, Relative Mobility and Absolute Mobility}

We now wish to further study the dependence of absolute mobility on its determinants: income growth, changes in income inequality and relative mobility. Fig. 4 presents three hypothetical scenarios that summarize the intuition for the effects of these determinants - absolute mobility increases with income growth, but decreases with increasing inequality and relative mobility.
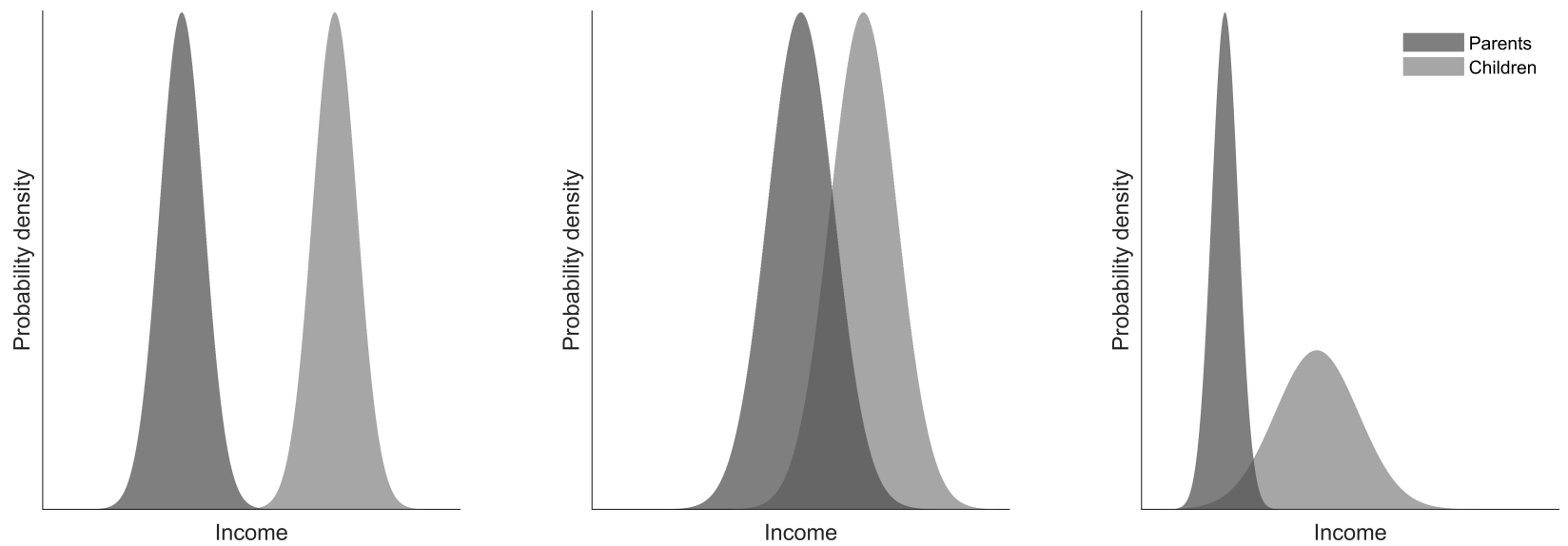

Figure 4: Descriptive scenarios of intergenerational changes in the income distribution. Left) No change in inequality between the two generations; growth is high enough so there is no overlap between the distributions, i.e. the poorest child is still richer than the richest parent. In this case absolute mobility will be $100 \%$ regardless of relative mobility; Middle) Inequality is still unchanged between the generations; growth is not as high as in the previous scenario. In this case the level of absolute mobility depends on relative mobility. In the extreme case of no relative mobility, absolute mobility will still be $100 \%$, since the richest parent is mapped into the richest child and so do the second richest parent and child and so on. Realistically, because relative mobility exists, absolute mobility will be lower than $100 \%$, and as relative mobility increases, absolute mobility would decrease; Right) Growth is as high as in the left panel, but inequality is higher among the children. This case is similar to the middle one - absolute mobility will be lower than $100 \%$ and as relative mobility increases, absolute mobility would decrease.

To formalize the intuition we present a simplified model for intergenerational mobility. Our starting point is $N$ parent-child pairs, and we denote by $Y_{p i}$ and $Y_{c i}$ the parents' and children's real incomes (at the same age) in family $i=1 \ldots N$, respectively. We assume that the incomes are positive and define the log-incomes $X_{p i}=\log Y_{p i}$ and $X_{c i}=\log Y_{c i}$.

A standard model for the intergenerational relationship between log-incomes is the following linear regression, in which the slope $(\beta)$ is the intergenerational earnings elasticity (IGE):

$$
X_{c i}=\alpha+\beta X_{p i}+\epsilon_{i} .
$$


Assuming that $\epsilon_{i}$ and $X_{p i}$ are normally distributed, so are $X_{c i}$. The marginal income distributions are then $\log$-normal $\left(Y_{p i} \sim \log \mathcal{N}\left(\mu_{p}, \sigma_{p}^{2}\right)\right.$ and $\left.Y_{c i} \sim \log \mathcal{N}\left(\mu_{c}, \sigma_{c}^{2}\right)\right)$. The joint parent-child $\log$-income distribution is a bivariate normal distribution, with correlation $\rho=\beta \frac{\sigma_{p}}{\sigma_{c}}$. The joint distribution is therefore fully specified by five parameters: $\mu_{p}, \sigma_{p}, \mu_{c}, \sigma_{c}$ and $\rho$.

These assumptions are standard (Pinkovskiy and Sala-i-Martin, 2009), and "the lognormal is a good approximation of empirical income distributions, leads to tractable results, and allows for an unambiguous definition of inequality." (Bénabou, 2000). As we demonstrate below, this model is also satisfactory for estimating the long run trend of absolute mobility.

The rate of absolute mobility, $A$, is the fraction of children earning more than their parents in real terms, equal to the probability $P\left(X_{c i}-X_{p i}>0\right)$. We can now derive a closed-form expression for absolute mobility in terms of the model parameters:

Proposition 1 For a bivariate log-normal distribution with parameters $\mu_{p}, \sigma_{p}$ (for the parents' marginal distribution), $\mu_{c}, \sigma_{c}$ (for the children's marginal distribution) and correlation $\rho$, the rate of absolute mobility is

$$
A=\Phi\left(\frac{\mu_{c}-\mu_{p}}{\sqrt{\sigma_{p}^{2}-2 \rho \sigma_{p} \sigma_{c}+\sigma_{c}^{2}}}\right),
$$

where $\Phi$ is the cumulative distribution function of the standard normal distribution, and (assuming $\left.\mu_{c}>\mu_{p}\right)$

$$
\frac{\partial A}{\partial \rho}>0
$$

To demonstrate that the model is empirically sound we use pre-tax national income per adult data in France to obtain $\mu_{p}, \sigma_{p}, \mu_{c}$ and $\sigma_{c}$ for every cohort and compare the baseline estimates in Section 3.2 to Eq. (4.2). We use France specifically for the fine-grained distributional data available since the early 20th century (Garbinti, Goupille-Lebret and Piketty, 2018).

In a log-normal distribution, income shares are uniquely determined by $\sigma,{ }^{5}$ so we estimate $\sigma$ for every year in four ways: based on the bottom $50 \%$ income share; the top $10 \%$ income share; the top $1 \%$ income share; the Gini coefficient. After obtaining $\sigma, \mu$ is given by $\mu=\log (m)-\frac{\sigma^{2}}{2}$, where $m$ is the per-adult pre-tax income given by data. The different ways to determine the value of $\sigma$ would correspond to four different series approximating the evolution of absolute mobility using Eq. (4.2). Figure 5 presents a comparison between the baseline absolute mobility estimates for France and the log-normal approximations. For the same rank correlation used in Section 3.2, the differences between the baseline estimate and the model are small. They average between -1.4 to 1.6 per-

\footnotetext{
${ }^{5}$ The share of top quantile $q$ in a log-normal distribution is $1-\Phi\left(\Phi^{-1}(1-q)-\sigma\right)$ and the Gini coefficient is $\operatorname{erf}\left(\frac{\sigma}{2}\right)$ (Cowell, 2011), where $\Phi$ is the cumulative distribution function of the standard normal distribution, and erf is the Gauss error function.
} 
centage points across the different approximations. Moreover, the long run trends in all cases are nearly identical, and the pairwise correlations between the series are all above 0.91. Fig. 5 demonstrates that despite its methodological naïvety, the bivariate log-normal model describes well the long run evolution of absolute mobility. Thus, it is also useful when very limited information on the evolution of the income distributions is available. Specifically, the knowledge of historical income growth rates and of either Gini coefficients or top $10 \%$ income shares is satisfactory for estimating absolute mobility.

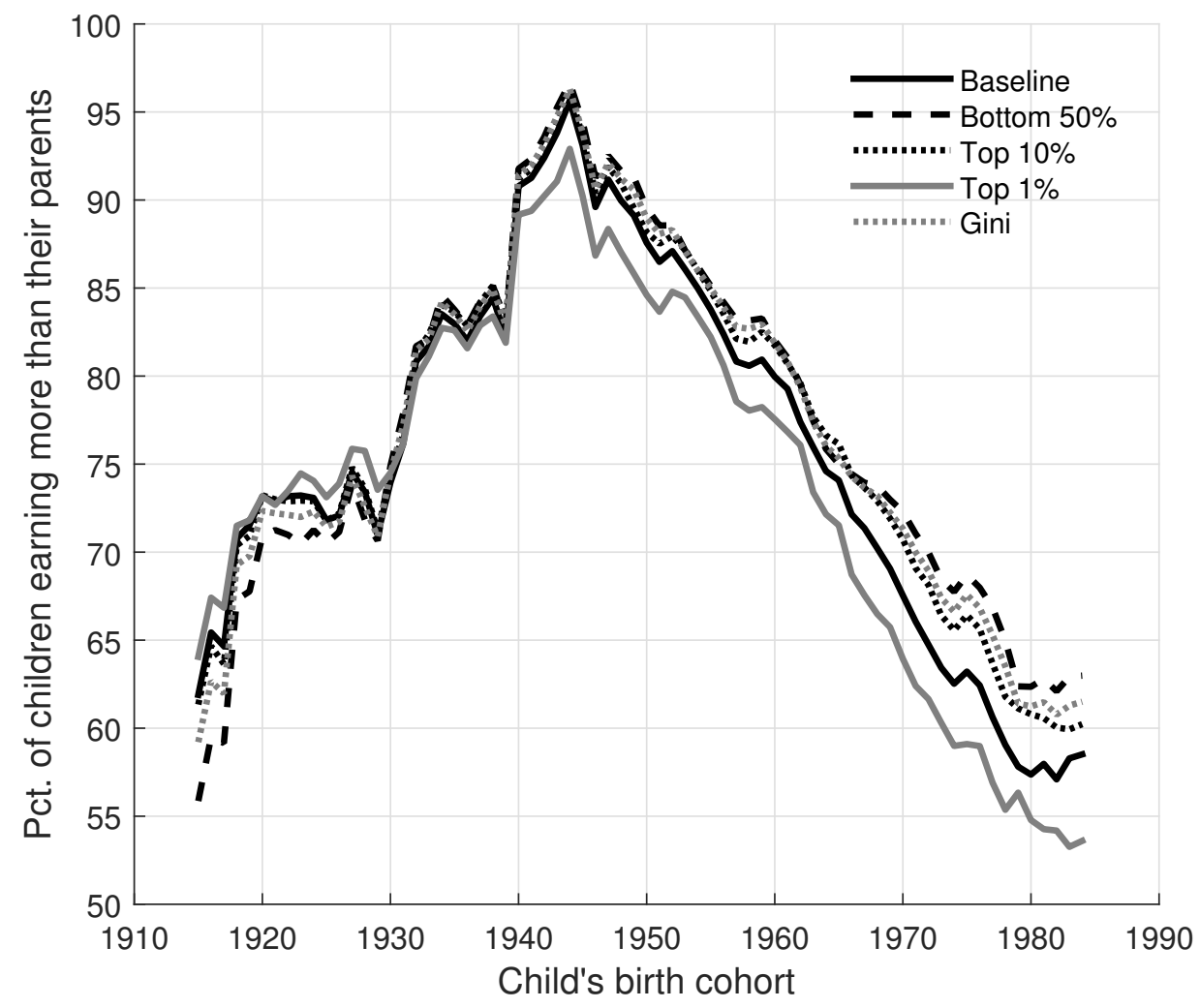

Figure 5: Absolute mobility in France under the bivariate log-normal approximation. The baseline estimates (solid black curve) are the same as in Fig. 2. In the other estimates each case is based on the bivariate log-normal approximation, with the value of $\sigma$ determined by different inequality measures - the bottom 50\% income share (dashed black); the top 10\% income share (dotted black); the top 1\% income share (solid gray); the Gini coefficient (dotted gray). The rank correlation was assumed as 0.3 in all cases.

Proposition 1 also shows that the bivariate log-normal model predicts an inverse relationship between absolute and relative mobility (assuming positive income growth between generations) - absolute mobility $(A)$ increases with the log-incomes correlation $(\rho)$. This seemingly counter-intuitive inverse relationship repeats the intuition illustrated in Fig. 4. It stands in contrast to the findings about absolute intergenerational mobility in Fig. 2 - the order of countries by either absolute or relative mobility is similar. However, as shown above, absolute mobility is driven primarily by economic growth, secondarily by changes in inequality, and only weakly by relative mobility. 
We note that Katz and Krueger (2017) discuss an alternative measure of absolute mobility which does not require panel data - the share of children earning more than the median parent. Based on Chetty et al. (2017), they find that this measure moves almost identically to the standard absolute mobility measure across birth cohorts in the United States. Using the simplified model we are able to show that this measure will be close to the rate of absolute mobility only if the IGE is close to $1 / 2$ (see Appendix J). Thus, it is no surprise that for the United States these measures are similar. Aaronson and Mazumder (2008) estimate the IGE for 1950-1970 birth cohorts in the United States at 0.46-0.58. In countries such as Canada or Denmark, in which the IGE is substantially lower than 0.5 (Corak, 2013), the Katz-Krueger measure of absolute mobility will lead to overestimation of absolute mobility. For example, in Denmark, the average estimated absolute mobility for $1950-1980$ birth cohorts is $78.5 \%$. For the Katz-Krueger mobility the average value is 87.2\%. In the United States this difference is much smaller: $62.2 \%$ and $63.7 \%$, respectively. Thus, the Katz-Krueger mobility cannot be used as a proxy for absolute mobility unless the IGE is close to $1 / 2$.

\section{Conclusion}

This paper highlights a decreasing absolute mobility trend in a large group of developed countries for post-World War II birth cohorts. Yet, the sources of this trend differ from country to country. In Australia and the United States, and to a lesser extent in Finland and the United Kingdom, the rising income inequality is the main contributor for decreasing absolute mobility. In other countries, a similar historic evolution of absolute mobility is predominantly explained by the decrease of income growth rates. We were also able to detect an increase in absolute intergenerational mobility in France, Sweden and the United States for pre-World War II birth cohorts. Our findings also extend those of Chetty et al. (2017). They indicate that the levels of absolute mobility were high even before World War II in the United States, and peaked around the 1940 birth cohort.

Our findings also imply that it is possible to produce estimates of absolute intergenerational mobility without the need for high-quality panel data sets. The basic structure of realistic copulas is similar, and therefore different measures of relative mobility are effectively interchangeable. This means that collapsing the copula into a single representative measure of relative mobility is empirically justified for estimating the rate of absolute mobility. Also, the sensitivity of the absolute mobility

to relative mobility is low. Thus, realistic changes in relative mobility cannot explain the evolution of absolute mobility. We also find that a bivariate log-normal model is satisfactory for describing the long run dynamics of absolute mobility. These findings allow to further study absolute mobility in more countries and additional birth cohorts in the absence of detailed historical panel data.

Our findings join recent work on global inequality and the effects of globalization (Bourguignon, 2015; Milanovic, 2016; Rodrik, 2017; Alvaredo et al., 2017; Ravallion, 2018). Some scholars link the recent global "populism wave" to globalization and inequality and their relationship (Milanovic, 
2016; Rodrik, 2017). Absolute intergenerational mobility captures this relationship. This can explain, for example, similarities in the recent political trends in France, the United Kingdom and the United States (see also Piketty (2018)). These countries have experienced the so-called "populism wave" during the recent presidential elections and the EU referendum in the United Kingdom. Inequality has been increasing during the past few decades in the United Kingdom and the United States. Yet, in France, inequality remained largely stable and at much lower levels. Gross domestic product growth, however, was almost consistently higher in the United States and in the United Kingdom than in France at the same period. Absolute intergenerational mobility captures an aspect of the chances of young adults to achieve a higher standard of living than their parents. It is low when growth is slow or when income inequality is very high (or both). Thus, the downward trend in absolute mobility demonstrates a possible partial explanation for the common political phenomena of the recent years.

Our methodology can be used to estimate not only absolute intergenerational mobility, but also intragenerational mobility. Many developed countries have gone through a recovery from a major crisis during the past decade. It has benefited most of the population, but not the entire population. In the United States, national income per adult increased in 13\% between 2009 and 2016. Yet, only about 55\% of the adults enjoyed a higher standard of living in 2016 than in 2009 (Berman and Bourguignon, 2019). A thorough analysis of intragenerational mobility is left for future work. 


\section{References}

Aaronson, Daniel, and Bhashkar Mazumder. 2008. "Intergenerational Economic Mobility in the United States, 1940 to 2000." Journal of Human Resources, 43(1): 139-172.

Alvaredo, Facundo, Anthony B. Atkinson, Lucas Chancel, Thomas Piketty, Emmanuel Saez, and Gabriel Zucman. 2016. "Distributional National Accounts (DINA) Guidelines: Concepts and Methods used in WID.world."

Alvaredo, Facundo, Lucas Chancel, Thomas Piketty, Emmanuel Saez, and Gabriel Zucman. 2017. "Global Inequality Dynamics: New Findings from WID.world." American Economic Review, 107(5): 404-409.

Atkinson, Anthony B. 1983. Social Justice and Public Policy. MIT press.

Atkinson, Anthony B., and François Bourguignon. 1982. "The Comparison of MultiDimensioned Distributions of Economic Status." The Review of Economic Studies, 183-201.

Bartholomew, David J. 1967. Stochastic Models for Social Processes. New York, NY:John Wiley \& Sons.

Becker, Gary S., and Nigel Tomes. 1979. "An Equilibrium Theory of the Distribution of Income and Intergenerational Mobility." Journal of Political Economy, 87(6): 1153-1189.

Bénabou, Roland. 2000. "Unequal Societies: Income Distribution and the Social Contract." American Economic Review, 90(1): 96-129.

Berman, Yonatan. 2019. "On the Link Between Intergenerational Mobility and Inequality: Are They Truly Distinct?" Mimeo.

Berman, Yonatan, and François Bourguignon. 2019. "Anonymous and Non-Anonymous Growth Incidence Curves: Evidence from the United States over the Last 50 Years." Mimeo.

Blanchet, Thomas, Juliette Fournier, and Thomas Piketty. 2017. "Generalized Pareto Curves: Theory and Applications."

Blanden, Jo, Stephen Machin, and Sumaiya Rahman. 2019. "Falling Real Wages: The Effect on Absolute Earnings Mobility." Mimeo.

Bonhomme, Stéphane, and Jean-Marc Robin. 2009. "Assessing the Equalizing Force of Mobility Using Short Panels: France, 1990-2000." The Review of Economic Studies, 76(1): 63-92.

Borjas, George J. 1992. "Ethnic Capital and Intergenerational Mobility." The Quarterly Journal of Economics, 107(1): 123-150.

Bourguignon, François. 2004. "The Poverty-Growth-Inequality Triangle." ICRIER Working paper 125 . 
Bourguignon, François. 2015. The Globalization of Inequality. Princeton University Press.

Bratberg, Espen, Øivind Anti Nilsen, and Kjell Vaage. 2005. "Intergenerational Earnings Mobility in Norway: Levels and Trends." Scandinavian Journal of Economics, 107(3): 419-435.

Breen, Richard, and David B. Rottman. 2014. Class Stratification: Comparative Perspectives. Routledge.

Breen, Richard, and Jan O. Jonsson. 2005. "Inequality of Opportunity in Comparative Perspective: Recent Research on Educational Attainment and Social Mobility." Annual Review of Sociology, 31: 223-243.

Chetty, Raj, David Grusky, Maximilian Hell, Nathaniel Hendren, Robert Manduca, and Jimmy Narang. 2017. "The Fading American Dream: Trends in Absolute Income Mobility Since 1940." Science, 356(6336): 398-406.

Chetty, Raj, Nathaniel Hendren, Patrick Kline, and Emmanuel Saez. 2014a. "Where is the Land of Opportunity? The Geography of Intergenerational Mobility in the United States." The Quarterly Journal of Economics, 129(4): 1553-1623.

Chetty, Raj, Nathaniel Hendren, Patrick Kline, Emmanuel Saez, and Nicholas Turner. 2014b. "Is the United States Still a Land of Opportunity? Recent Trends in Intergenerational Mobility." American Economic Review, 104(5): 141-147.

Corak, Miles. 2013. "Income Inequality, Equality of Opportunity, and Intergenerational Mobility." Journal of Economic Perspectives, 27(3): 79-102.

Corak, Miles, Matthew J. Lindquist, and Bhashkar Mazumder. 2014. "A Comparison of Upward and Downward Intergenerational Mobility in Canada, Sweden and the United States." Labour Economics, 30: 185-200.

Cowell, Frank. 2011. Measuring Inequality. Oxford, UK:OUP Oxford.

Eberharter, Veronika V. 2014. "The Intergenerational Dynamics of Social Inequality - Empirical Evidence from Europe and the United States." Global Journal of Human-Social Science: E, 14(1): 95-108.

Erikson, Robert, and John H. Goldthorpe. 1992. The Constant Flux: A Study of Class Mobility in Industrial Societies. Oxford University Press, USA.

Erikson, Robert, John H. Goldthorpe, and Lucienne Portocarero. 1979. "Intergenerational Class Mobility in Three Western European Societies: England, France and Sweden." The British Journal of Sociology, 30(4): 415-441.

Eurostat. 2018. "Household Composition Statistics." Accessed: 07/05/2018. 
Garbinti, Bertrand, Jonathan Goupille-Lebret, and Thomas Piketty. 2018. "Income Inequality in France, 1900-2014: Evidence from Distributional National Accounts (DINA)." Journal of Public Economics, 162: 63-77.

Goldthorpe, John H. 1987. Social Mobility and Class Structure in Modern Britain. Oxford, UK:Oxford University Press.

Jäntti, Markus, Bernt Bratsberg, Knut Roed, Oddbjorn Raaum, Robin Naylor, Eva Österbacka, Anders Björklund, and Tor Eriksson. 2006. "American Exceptionalism in a New Light: A Comparison of Intergenerational Earnings Mobility in the Nordic Countries, the United Kingdom and the United States." IZA Discussion Paper 1938.

Katz, Lawrence F., and Alan B. Krueger. 2017. "Documenting Decline in US Economic Mobility." Science, 356(6336): 382-383.

Lee, Chul-In, and Gary Solon. 2009. "Trends in Intergenerational Income Mobility." The Review of Economics and Statistics, 91(4): 766-772.

Lefranc, Arnaud, and Alain Trannoy. 2005. "Intergenerational Earnings Mobility in France: Is France More Mobile than the US?" Annales d'Economie et de Statistique, 57-77.

Leigh, Andrew. 2007. "Intergenerational Mobility in Australia." The BE Journal of Economic Analysis \& Policy, 7(2).

Lipset, Seymour M., and Hans L. Zetterberg. 1959. "Social Mobility in Industrial Societies." In Social Mobility in Industrial Society. , ed. Seymour M. Lipset and Reinhard Bendix. University of California Press Berkeley, CA.

Lipset, Seymour M., and Natalie Rogoff. 1954. "Class and Opportunity in Europe and America." Commentary, 17: 562-568.

Liss, Erik, Martin Korpi, and Karl Wennberg. 2019. "The American Dream Lives in Sweden: Trends in Intergenerational Absolute Income Mobility.” Ratio Working Paper No. 325.

Manduca, Robert, Maximilian Hell, Adrian Adermon, Jo Blanden, Espen Bratberg, Keun Bok Lee, Stephen Machin, Martin D. Munk, Martin Nybom, Yuri Ostrovsky, Sumaiya Rahman, and Outi Sirniö. 2019. "Trends in Absolute Income Mobility in North America and Europe." Mimeo.

Mazumder, Bhashkar. 2005. "Fortunate Sons: New Estimates of Intergenerational Mobility in the United States Using Social Security Earnings Data." Review of Economics and Statistics, $87(2): 235-255$.

Milanovic, Branko. 2016. Global Inequality: A New Approach for the Age of Globalization. Cambridge, MA:Harvard University Press. 
Obama, Barack H. 2013. "Remarks by the President on Economic Mobility."

Ostrovsky, Yuri. 2017. "Doing as Well as One's Parents?: Tracking Recent Changes in Absolute Income Mobility in Canada." Statistics Canada.

Ostry, Jonathan D., Andrew Berg, and Charalambos G. Tsangarides. 2014. "Redistribution, Inequality, and Growth." International Monetary Fund.

Piketty, Thomas. 2018. "Brahmin Left vs Merchant Right: Rising Inequality and the Changing Structure of Political Conflict." Mimeo.

Piketty, Thomas, and Gabriel Zucman. 2014. "Capital is Back: Wealth-Income Ratios in Rich Countries, 1700-2010." The Quarterly Journal of Economics, 129(3): 1255-1310.

Piketty, Thomas, Emmanuel Saez, and Gabriel Zucman. 2018. "Distributional National Accounts: Methods and Estimates for the United States." The Quarterly Journal of Economics, 133(2): 553-609.

Pinkovskiy, Maxim, and Xavier Sala-i-Martin. 2009. "Parametric Estimations of the World Distribution of Income." Working Paper 15433, NBER.

Plackett, Robin L. 1965. "A Class of Bivariate Distributions." Journal of the American Statistical Association, 60(310): 516-522.

Ravallion, Martin. 2018. "Inequality and Globalization: A Review Essay." Journal of Economic Literature, 56(2): 620-42.

Rodrik, Dani. 2017. "Populism and the Economics of Globalization." National Bureau of Economic Research.

Shorrocks, Anthony F. 1978. "The Measurement of Mobility." Econometrica: Journal of the Econometric Society, 46(5): 1013-1024.

Solon, Gary. 1992. "Intergenerational Income Mobility in the United States." American Economic Review, 82(3): 393-408.

Spearman, Charles. 1904. "The Proof and Measurement of Association between Two Things." The American Journal of Psychology, 15(1): 72-101.

The World Inequality Database. 2018. "Canada, Denmark, Finland, France, Norway, Sweden, UK and USA per Adult National Income." http://wid.world/data/.

Trivedi, Pravin K., and David M. Zimmer. 2007. "Copula Modeling: An Introduction for Practitioners." Foundations and Trends in Econometrics, 1(1): 1-111.

Ueda, Atsuko. 2009. "Intergenerational Mobility of Earnings and Income in Japan." The BE Journal of Economic Analysis \& Policy, 9(1). 
United States Bureau of Labor Statistics. 2017. "Current Population Survey Income Data." Accessed: 07/05/2017. 


\section{A Proofs}

\section{A.1 Proof of proposition 1}

For a bivariate log-normal distribution with parameters $\mu_{p}, \sigma_{p}$ (for the parents' marginal distribution), $\mu_{c}, \sigma_{c}$ (for the children's marginal distribution) and correlation $\rho$, the rate of absolute mobility is

$$
A=\Phi\left(\frac{\mu_{c}-\mu_{p}}{\sqrt{\sigma_{p}^{2}-2 \rho \sigma_{p} \sigma_{c}+\sigma_{c}^{2}}}\right),
$$

where $\Phi$ is the cumulative distribution function of the standard normal distribution, and (assuming $\left.\mu_{c}>\mu_{p}\right)$

$$
\frac{\partial A}{\partial \rho}>0
$$

Proof: First, by definition, the correlation $\rho$, between $X_{p}$ and $X_{c}$ equals to their covariance, divided by $\sigma_{p} \sigma_{c}$

$$
\rho=\frac{\operatorname{Cov}\left[X_{p}, X_{c}\right]}{\sigma_{p} \sigma_{c}}
$$

$\beta$ can be directly calculated as follows, by the linear regression slope definition:

$$
\beta=\frac{\sum_{i=1}^{N}\left(X_{p}^{i}-\bar{X}_{p}\right)\left(X_{c}^{i}-\bar{X}_{c}\right)}{\sum_{i=1}^{N}\left(X_{p}^{i}-\bar{X}_{p}\right)},
$$

where $\bar{X}_{p}$ and $\bar{X}_{c}$ are the average parent and child log-incomes, respectively.

It follows that

$$
\beta=\frac{\operatorname{Cov}\left[X_{p}, X_{c}\right]}{\sigma_{p}^{2}}
$$

We immediately obtain

$$
\beta=\frac{\sigma_{c}}{\sigma_{p}} \rho
$$

Now we define a new random variable $Z=X_{c}-X_{p}$. It follows that calculating $A$ is equivalent to calculating the probability $P(Z>0)$.

Subtracting two dependent normal distributions yields

$$
Z \sim \mathcal{N}\left(\mu_{c}-\mu_{p}, \sigma_{p}^{2}+\sigma_{c}^{2}-2 \operatorname{Cov}\left[X_{p}, X_{c}\right]\right)
$$


and it follows, due to Eq. (A.6), that

$$
Z \sim \mathcal{N}\left(\mu_{c}-\mu_{p}, \sigma_{p}^{2}(1-2 \beta)+\sigma_{c}^{2}\right)
$$

If follows that

$$
\frac{Z-\left(\mu_{c}-\mu_{p}\right)}{\sqrt{\sigma_{p}^{2}(1-2 \beta)+\sigma_{c}^{2}}} \sim \mathcal{N}(0,1)
$$

so we can now write

$$
\begin{aligned}
& P(Z>0)= \\
& P\left(\frac{Z-\left(\mu_{c}-\mu_{p}\right)}{\sqrt{\sigma_{p}^{2}(1-2 \beta)+\sigma_{c}^{2}}}>-\frac{\mu_{c}-\mu_{p}}{\sqrt{\sigma_{p}^{2}(1-2 \beta)+\sigma_{c}^{2}}}\right)= \\
& \Phi\left(\frac{\mu_{c}-\mu_{p}}{\sqrt{\sigma_{p}^{2}(1-2 \beta)+\sigma_{c}^{2}}}\right)=\Phi\left(\frac{\mu_{c}-\mu_{p}}{\sqrt{\sigma_{p}^{2}-2 \rho \sigma_{p} \sigma_{c}+\sigma_{c}^{2}}}\right),
\end{aligned}
$$

where $\Phi$ is the cumulative distribution function of the standard normal distribution.

Taking the partial derivative of this expression with respect to the correlation $\rho$ we get

$$
\frac{\partial A}{\partial \rho}=\frac{\left(\mu_{c}-\mu_{p}\right) \sigma_{p} \sigma_{c} e^{-\frac{\left(\mu_{c}-\mu_{p}\right)^{2}}{2\left(\sigma_{p}^{2}-2 \rho \sigma_{p} \sigma_{c}+\sigma_{c}^{2}\right)}}}{\sqrt{2 \pi}\left(\sigma_{p}^{2}-2 \rho \sigma_{p} \sigma_{c}+\sigma_{c}^{2}\right)^{3 / 2}} .
$$

Assuming $\mu_{c}>\mu_{p}$, it follows that $\frac{\partial A}{\partial \rho}>0$. 


\section{B Empirical Copulas and Measures of Relative Mobility}

In Fig. 1 we use copulas measured for different birth cohorts, different countries and for both pre-tax and post-tax incomes and compare them in terms of different measures of relative mobility. Our aim was to demonstrate that although relative mobility is measured by theoretically distinct measures, in practice, differences in one measure translate into proportional changes in other measures. These measures of relative mobility are effectively interchangeable.

Here we present in detail the different measures used for the comparison. We consider copulas as transition (doubly stochastic) matrices $P \in \mathscr{P}(N)$, where $p_{i j}$ represents the probability of transferring to quantile $j$ (child) for those starting in quantile $i$ (parent) and $N$ is the number of income quantiles. We consider four standard measures of relative mobility:

- Spearman's rank correlation (Spearman, 1904) (or rank-rank slope, $R R S$ ), defined as

$$
\rho_{S}(P)=\frac{12 \sum_{i=1}^{N} \sum_{j=1}^{N} i j p_{i j}-3 N(N+1)^{2}}{N\left(N^{2}-1\right)}
$$

- Bartholomew's index (Bartholomew, 1967) (average absolute jump), defined as

$$
B(P)=\frac{1}{N} \sum_{i=1}^{N} \sum_{j=1}^{N}|i-j| p_{i j}
$$

- Average absolute non-zero jump, defined as the average absolute jump while excluding the trace of $P$, or

$$
N Z(P)=\frac{N \cdot B(P)}{N-\operatorname{tr}(P)}
$$

- Shorrocks' trace index (Shorrocks, 1978), defined as

$$
S(P)=\frac{N-\operatorname{tr}(P)}{N-1}
$$

The different measures are mathematically related, however they are not linearly dependent. Specifically, it is possible to construct matrices which have the same trace index, but very different rank correlation, average absolute non-zero jump measure or Bartholomew's index and vice versa. Bartholomew (1967); Shorrocks (1978); Atkinson and Bourguignon (1982); Atkinson (1983) provide several constructive examples demonstrating the differences between such measures. They describe mathematical constructions of copulas such that one measure is preserved while others may change.

For the comparison in Fig. 1 we used 28 transition matrices estimated using survey and tax data covering pre-tax incomes in Denmark, Finland, Norway, Sweden, the United Kingdom and the 
United States (Jäntti et al., 2006) for different birth cohorts in each, estimated for fathers and daughters and for fathers and sons; post-tax incomes in Germany, the United Kingdom and the United States (Eberharter, 2014); de-identified federal income tax returns for pre-tax incomes in the United States (Chetty et al., 2014a). Fig. 1 demonstrates that despite the a priori independence of the relative mobility measures, they are, in fact, almost linearly related across time and countries, both for pre- and post-tax incomes. We conclude, therefore, that the shape of the copulas is similar and they can be practically summarized by a single parameter. We use the rank correlation since in many countries the estimated relative mobility is simply reported using the rank correlation or the intergenerational elasticity (from which the rank correlation can be deduced), rather than providing the entire copula. Also, the rank correlation is more empirically robust than other measures (Chetty et al., 2014a). 


\section{Sensitivity of Absolute Mobility to Fixed Rank Correlation}

In our estimates we use Spearman's rank correlation (or rank-rank slope) to describe the copula between the income distributions of parents and children. We then continue to estimate the absolute intergenerational mobility. We assume that given the marginal distributions, the rank correlation determines absolute mobility. Conceptually, the same rank correlation could deliver very different absolute mobility estimates. Yet, as we describe below, this requires the copulas to be unrealistic. We consider copulas as transition (doubly stochastic) matrices $P \in \mathscr{P}(N)$, where $p_{i j}$ represents the probability of transferring to quantile $j$ (child) for those starting in quantile $i$ (parent) and $N$ is the number of income quantiles. Evidence shows that the diagonal elements are generally higher and the transition probabilities decrease with the transition distance. The probability to move between two ranks $i$ and $j$ within two generations is a decreasing function of $|i-j|$ (see, for example Jäntti et al. (2006); Chetty et al. (2017)). Preserving the rank correlation, while creating a large effect on absolute mobility requires breaking this regularity.

The rank correlation of a transition matrix is

$$
\rho_{S}(P)=\frac{12 \sum_{i=1}^{N} \sum_{j=1}^{N} i j p_{i j}-3 N(N+1)^{2}}{N\left(N^{2}-1\right)},
$$

thus, only the sum $\sum_{i=1}^{N} \sum_{j=1}^{N} i j p_{i j}$ depends on the matrix elements.

We now define a $\Delta$-local rank-correlation preserving move as a change to 8 elements in the matrix $-p_{i_{1}, j_{1}}, p_{i_{1}+1, j_{1}}, p_{i_{1}+1, j_{1}}, p_{i_{1}+1, j_{1}+1}$ and $p_{i_{2}, j_{2}}, p_{i_{2}+1, j_{2}}, p_{i_{2}+1, j_{2}}, p_{i_{2}+1, j_{2}+1}$ in the following way:

$$
\begin{aligned}
& p_{i_{1}, j_{1}} \rightarrow p_{i_{1}, j_{1}}+\Delta \\
& p_{i_{1}+1, j_{1}+1} \rightarrow p_{i_{1}+1, j_{1}+1}+\Delta \\
& p_{i_{1}+1, j_{1}} \rightarrow p_{i_{1}+1, j_{1}}-\Delta \\
& p_{i_{1}, j_{1}+1} \rightarrow p_{i_{1}, j_{1}+1}-\Delta \\
& p_{i_{2}, j_{2}} \rightarrow p_{i_{2}, j_{2}}-\Delta \\
& p_{i_{2}+1, j_{2}+1} \rightarrow p_{i_{2}+1, j_{2}+1}-\Delta \\
& p_{i_{2}+1, j_{2}} \rightarrow p_{i_{2}+1, j_{2}}+\Delta \\
& p_{i_{2}, j_{2}+1} \rightarrow p_{i_{2}, j_{2}+1}+\Delta
\end{aligned}
$$

where $\Delta$ can be either positive or negative (as long as all the elements remain non-negative and not greater than 1 ) and $i_{1}, j_{1}, i_{2}$ and $j_{2}$ can be any quantiles between 1 and $N-1$.

Such a change trivially preserves the sum $\sum_{i=1}^{N} \sum_{j=1}^{N} i j p_{i j}$ and therefore the rank correlation. By composing many $\Delta$-local rank-correlation preserving moves it is possible to change a given copula while preserving the rank correlation.

In general, rank-correlation preserving moves have the effect of increasing the trace while also 
increasing the extreme ends of the transition matrix, or vice versa. This is demonstrated in the three copulas in Fig. 6. They all share the same rank correlation (0.3), but are very different from one another. The copulas were constructed by composing several $\Delta$-local rank-correlation preserving moves on copula A, which is the copula used for producing the baseline estimates of absolute mobility in France. Only copula A is realistic and has the typical form of the empirical copulas (compare with Jäntti et al. (2006); Eberharter (2014)). Copula C is far from being plausible, attaching very high probabilities to the diagonal, zeros to some off diagonal elements, but non-zero probability to make the largest possible moves within generations.

Nevertheless, copulas A and B produce almost the same absolute mobility. This was tested for France, using the same marginal distributions used for the baseline estimates. For each of the three copulas we produced a series of estimated absolute mobility values. The results are also presented in Fig. 6. As expected, copula $\mathrm{C}$ leads to results that are different from the baseline estimates (copula A). Yet, the trend remains very similar to the baseline estimates and the results are 3.3 percentage points higher than the baseline estimate on average. Copula B leads to results that are almost identical to the baseline. This is regardless of it being unrealistic.

Together with the robustness of absolute mobility to different copula models (see Appendix D), our results indicate that for empirical copulas or standard copula models such as the Gaussian, Clayton, Gumbel or Plackett models, using the rank correlation as a single parameter describing the copula does not introduce any significant uncertainty or bias to the absolute mobility estimates. 

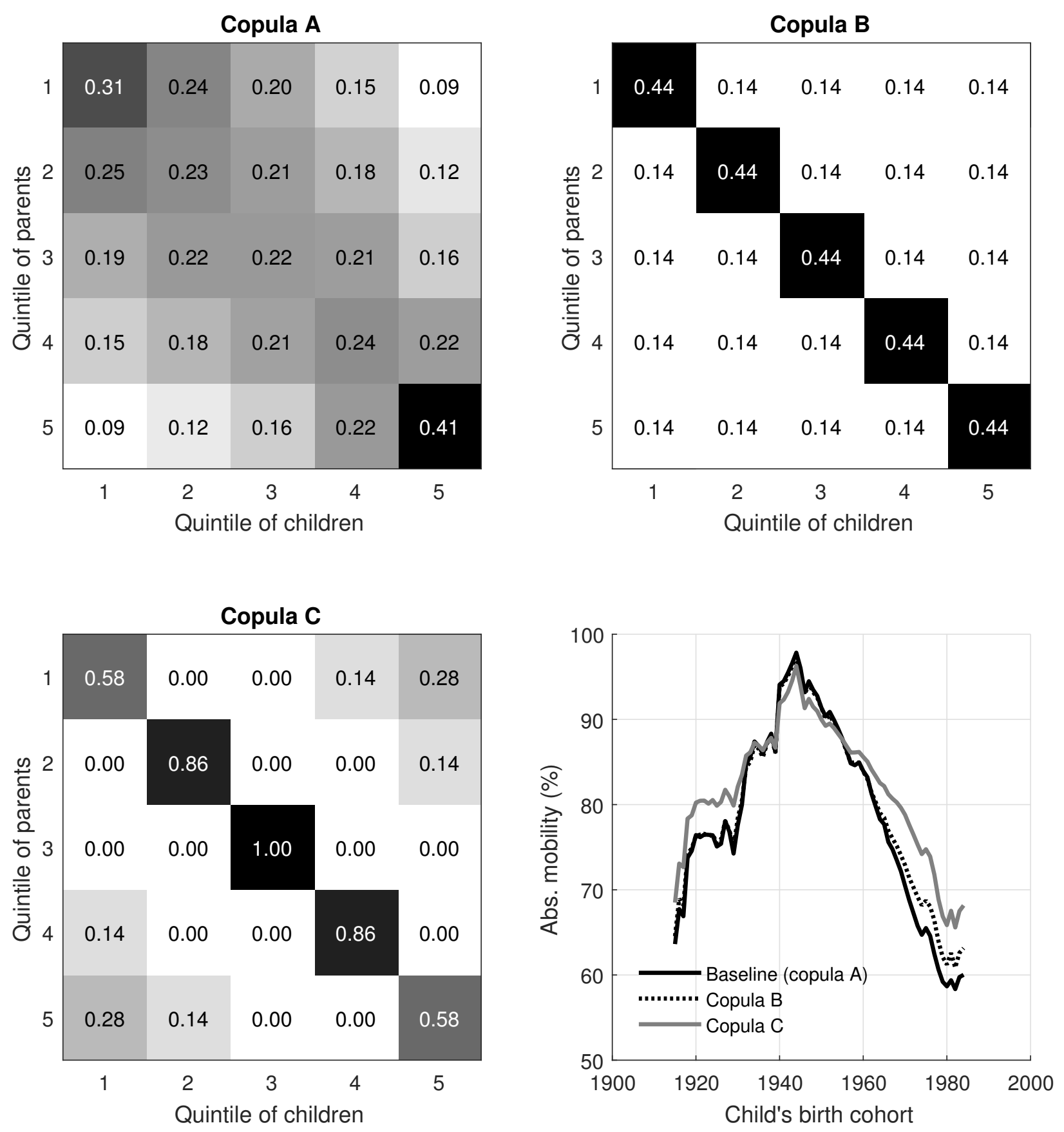

Figure 6: Three copulas (transition matrices) constructed by composing many $\Delta$-local rankcorrelation preserving moves. Copula $\mathrm{A}$ is similar to the copula used for producing the baseline estimates of absolute mobility in France. The bottom right panel shows the absolute mobility estimates for France when using the different copulas. 


\section{Log-Normal Model Sensitivity to the Copula Model}

The choice of copula model may affect the estimated absolute mobility in the methodology presented. We demonstrate that as long as the rank correlation is the same, the copula model effect on estimated absolute mobility is, in practice, insignificant. We compare four copula models - Gaussian, which is the copula in the bivariate log-normal model, as well as the Clayton, the Gumbel and the Plackett copula families (Plackett, 1965; Trivedi and Zimmer, 2007). In their study of relative intragenerational mobility in France, Bonhomme and Robin (2009) argue that the Gaussian copula "tends to underestimate the dependence in the middle of the distribution, that is, the probabilities of remaining in the second, third, and fourth quintiles" and show that the empirical copula is best estimated by the Plackett copula.

Figure 7 demonstrates that the differences between the absolute mobility estimates when using different copula models, while assuming the same rank correlations, are negligible. The average difference between each of the time series was less than 1 percentage point, i.e. an effect of less than $2 \%$.

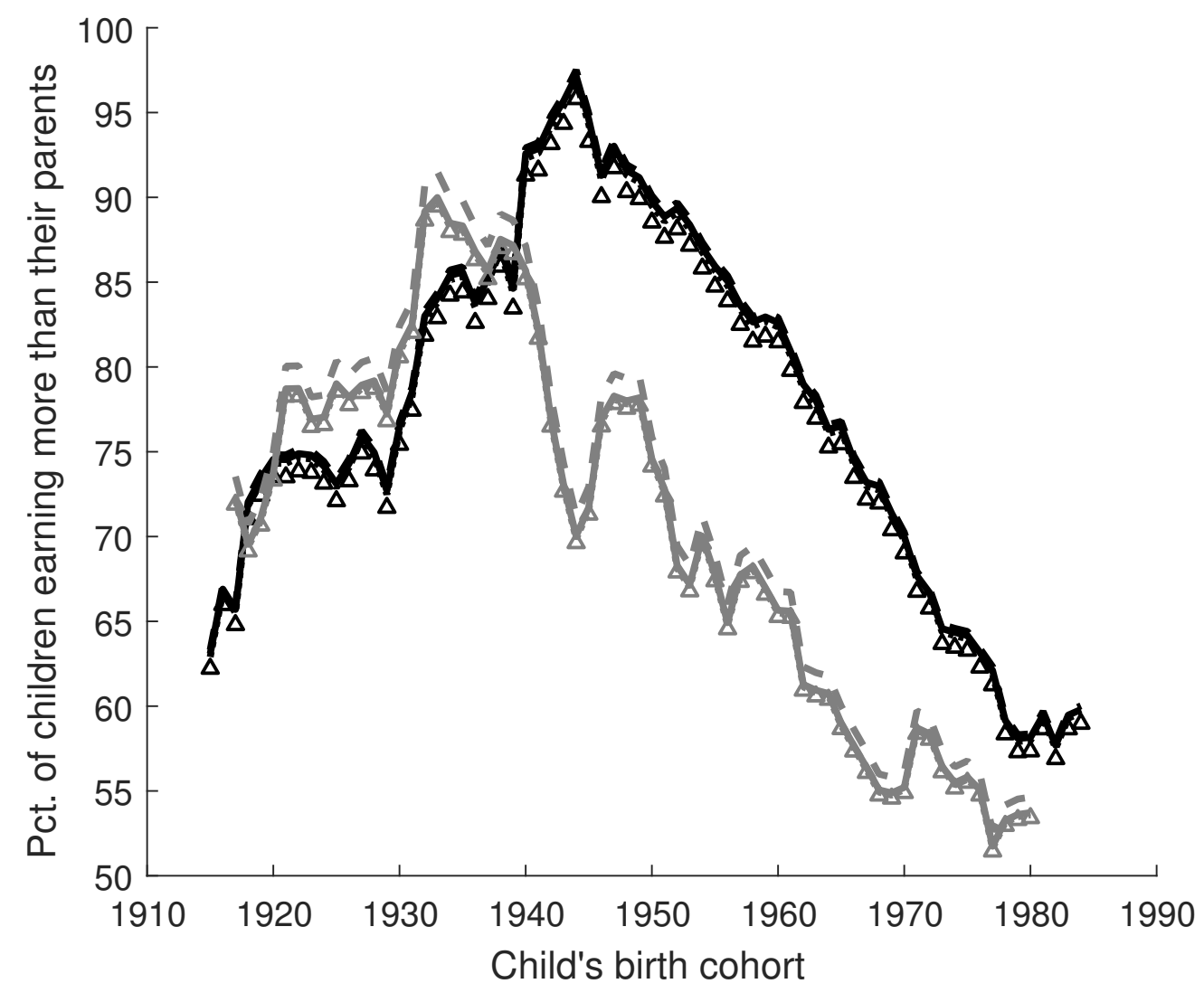

Figure 7: The copula model effect on the absolute mobility in France (black) and the United States (gray). The copula models used were Gaussian (solid lines), Clayton (dashed), Gumbel (dotted) and Plackett (triangles). 


\section{E Potential Effect of Individual and Family Incomes Differences on Absolute Mobility}

In order to make our analysis results comparable to those in Chetty et al. (2017) we use equal-split adults as the unit of observation of our income data when possible. In several countries, the income data are based, however, on individual or tax unit incomes. Tax units may be either individuals or families, depending on the country and the year. In some countries taxes are declared on an individual basis today, but not in the past.

In order to keep the method as simple as possible, and since this has only a small effect on the results, the baseline estimates do not take into account those differences. In Fig. 8 we show that under very conservative assumptions, ignoring the differences between individual and family income may lead to a downward bias of 5-6.5 percentage points in absolute intergenerational mobility, but would not change at all the trend.

The calculation assumes that the samples of individual incomes are randomly divided into two subsamples of equal size $-\left\{A_{i}\right\}$ and $\left\{B_{i}\right\}$. These sub-samples are then matched assuming a Gaussian copula with rank correlation of zero (meaning perfectly random matching) so that for a specific index $j, A_{j}$ corresponds to $B_{j}$. These represent spouses in a family and we assume that each family is composed of two spouses exactly. The matched incomes are then summed to create a new sample $\left\{C_{i}\right\}$, for $C_{i}=A_{i}+B_{i}$. This is done for every year and then absolute mobility is estimated the same way as the baseline estimate but assuming the $\left\{C_{i}\right\}$ samples rather than the original samples, based on individual incomes.

This is a conservative estimate since it ignores assortative mating. Assortative mating effectively increases the rank correlation between spouses' incomes, which we assume is $0 .{ }^{6}$ For a rank correlation of 1 , the absolute mobility estimates will be similar to those obtained without the sample splitting. The effect of sample splitting would increase with decreasing rank correlation. In addition, we assume that all families have two spouses and we ignore single-person families, for which individual data reflect family data. For these reasons, the difference between individual, equal-split and family income absolute mobility estimates is practically small.

In Denmark, Norway and Sweden, in which the data we use for absolute mobility estimates are individualized, $40 \%-50 \%$ of households are single-person families (Eurostat, 2018). We consider, in addition to the zero rank correlation conservative estimate, a more realistic estimate for these countries - we assume that for each of the individual income samples half remains unchanged and the other half is divided and matched in the way explained, assuming a rank correlation of 0.3 with Gaussian copula. Fig. 8 also presents these estimates, which are very close to the baseline estimates.

\footnotetext{
${ }^{6}$ Based on the CPS data (United States Bureau of Labor Statistics, 2017), the income rank correlation between spouses in the United States is 0.3 and was very stable from 1964 onward.
} 

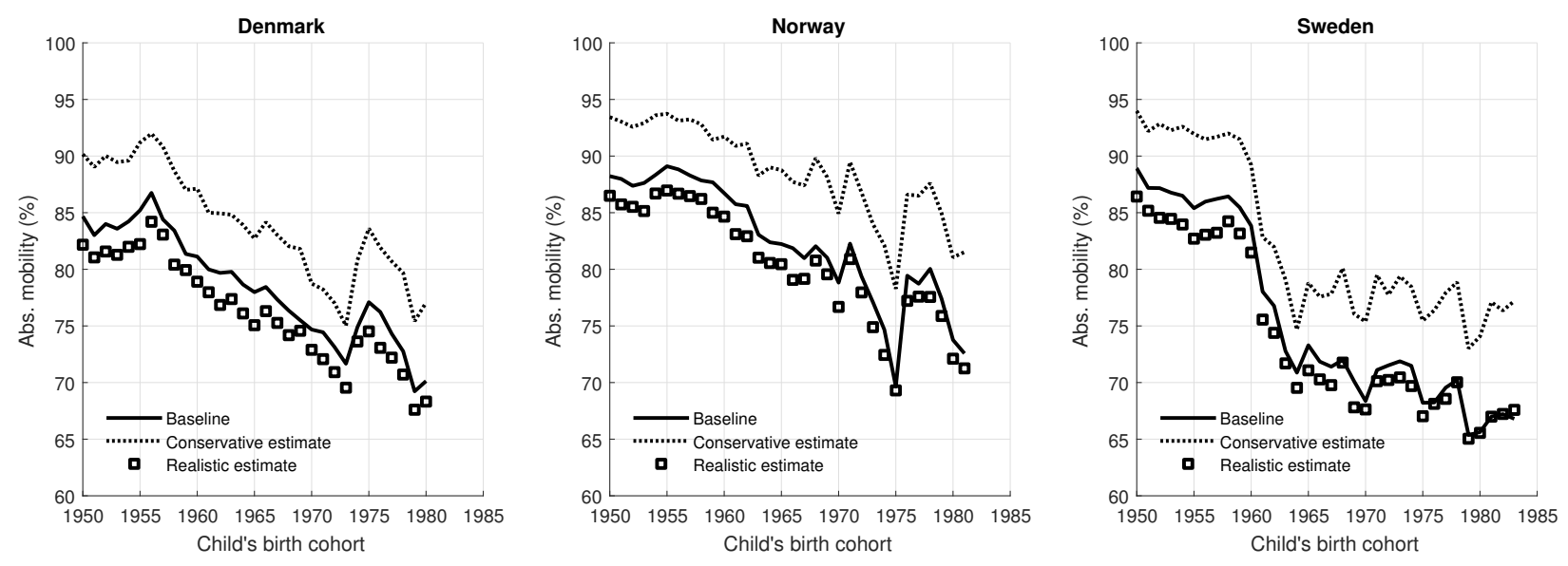

Figure 8: The absolute intergenerational mobility in Denmark, Norway and Sweden implementing assortative mating on individual income data. 


\section{F Data Specification}

The data used for producing the marginal income distributions are taken from The World Inequality Database (2018). We consider only years in which data were sufficiently detailed to use the generalized Pareto curve interpolation method (Blanchet, Fournier and Piketty, 2017). Hence the data used are available for the following countries and time periods:

Table 1: The availability of the income distribution for developed countries in The World Inequality Database (2018)

\begin{tabular}{lc}
\hline Country & Time period \\
\hline Australia & $1950-2016$ \\
Canada & $1950-2010$ \\
Denmark & $1950-2010$ \\
Finland & $1950-2009$ \\
France & $1915-2014$ \\
Japan & $1947-2010$ \\
Norway & $1950-2011$ \\
Sweden & $1903-2013$ \\
United Kingdom & $1959-2014$ \\
United States & $1917-2014$ \\
\hline
\end{tabular}

Data for the rank correlation in each of the above countries were taken from different studies, as are presented in Tab. 2. As noted, for the countries in which the interegenerational elasticity was reported, rather than the rank correlation, we use the relationship $\rho=\frac{\sigma_{p}}{\sigma_{c}} \beta$, where $\sigma_{p}$ and $\sigma_{c}$ are the standard deviations of the parent and child marginal income distributions and $\beta$ is the estimated intergenerational income elasticity. The rank correlation is approximated by $\rho_{S} \approx \frac{6 \arcsin (\rho / 2)}{\pi}$ (see Trivedi and Zimmer (2007)).

Table 2: Rank correlation values used in the absolute mobility analysis

\begin{tabular}{lcl}
\hline Country & Rank correlation & Source \\
\hline Australia & 0.22 & Leigh (2007) \\
Canada & 0.27 & Corak, Lindquist and Mazumder (2014) \\
Denmark & 0.19 & Jäntti et al. (2006) \\
Finland & 0.19 & Jäntti et al. (2006) \\
France & 0.30 & Lefranc and Trannoy (2005) \\
Japan & 0.30 & Ueda (2009) \\
Norway & 0.21 & Bratberg, Anti Nilsen and Vaage (2005) \\
Sweden & 0.20 & Jäntti et al. (2006) \\
United Kingdom & 0.30 & Jäntti et al. (2006) \\
United States & 0.30 & Chetty et al. (2014a्) \\
\hline
\end{tabular}




\section{G Absolute Intergenerational Mobility for the Entire Population and for Age Groups}

Garbinti, Goupille-Lebret and Piketty (2018) "combine national accounts, tax and survey data in a comprehensive and consistent manner to build homogeneous annual series on the distribution of national income by percentiles over the 1900-2014 period, with detailed breakdown by age, gender and income categories over the 1970-2014 period." Using the tabulated age-grouped data, it is possible to estimate the absolute intergenerational mobility in France for 1970, 1975 and 1979 cohorts by considering the income distribution of adults aged 20-39 in 1970, 1975 and 1979 as parents and in 2000, 2005 and 2009 as children. Assuming rank correlation of 0.3, the same as in the baseline estimates, we find that the estimates assuming 20-39 year-old adults, are lower than the baseline estimates by $2-5$ percentage points. This difference is not statistically significant, due to the large statistical error associated with the tabulated data. In addition, we can compare our baseline estimates for the United States to those reported in Chetty et al. (2017). The latter estimates are only based on samples of 30 year olds, rather than pooling all ages together as done for the baseline estimates.

The results are presented in Fig. 9. They illustrate the high similarity between the different estimates. Although pooling all ages together does change the results, this has only a small effect on the long run evolution of absolute mobility. 


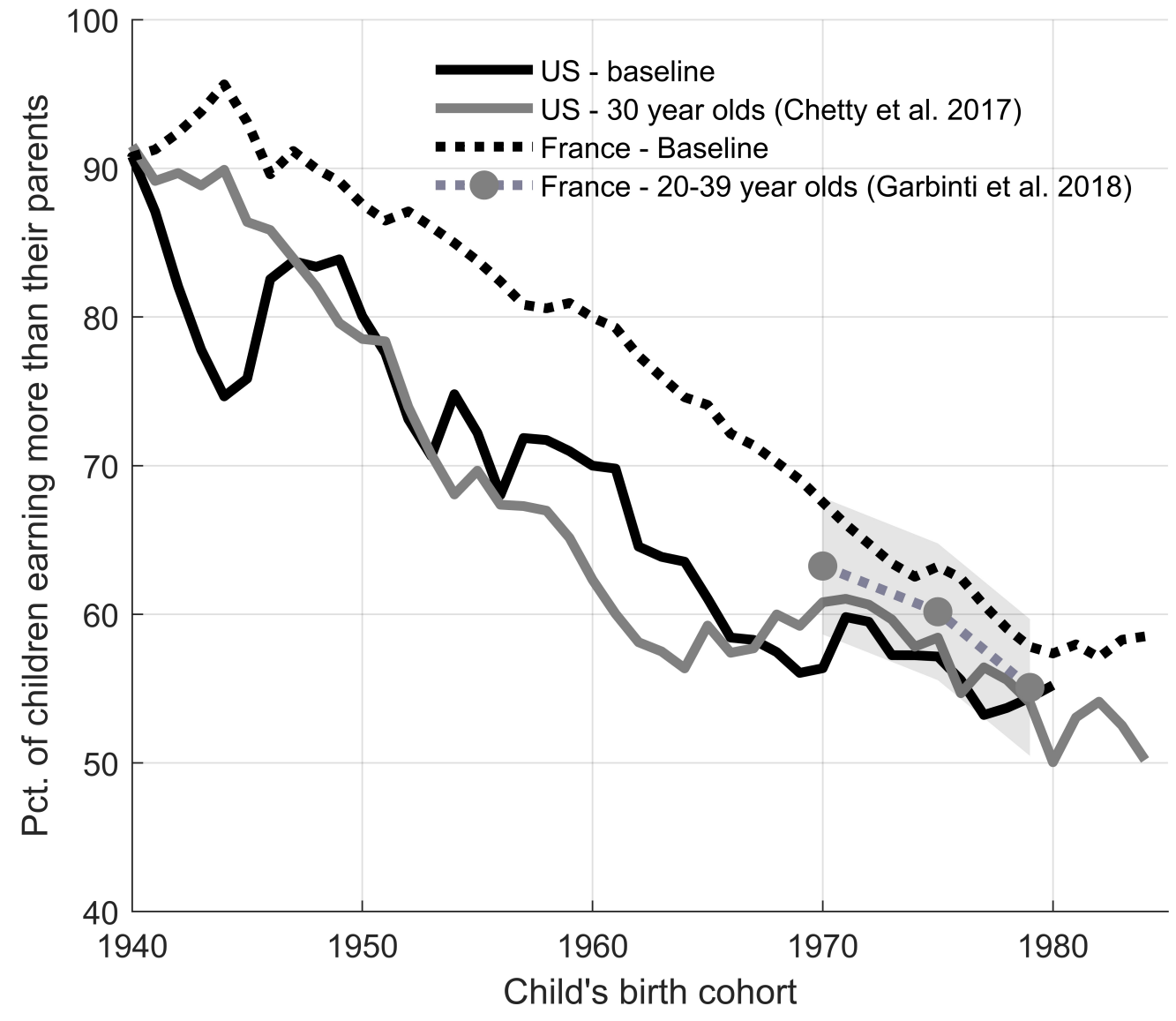

Figure 9: The absolute intergenerational mobility in the United States and in France for pooled ages and different age groups. The shaded gray areas represents $95 \%$ confidence intervals for the estimates produced by bootstrapping for France, based on the tabulations in Garbinti, GoupilleLebret and Piketty (2018). 


\section{H Detailed Results for the Evolution of Absolute Mobility}

The following tables present the absolute mobility estimates for a large group of developed countries and the results of the decomposition of the absolute mobility trends in different countries to the contributions of changes in inequality and income growth. These are presented in Fig. 2 and Fig. 3, respectively.

Table 3: The evolution of absolute intergenerational mobility in developed economies (see Fig. 2)

\begin{tabular}{|c|c|c|c|c|c|c|c|c|c|c|}
\hline Cohort & Australia & Canada & Denmark & Finland & France & Japan & Norway & Sweden & UK & US \\
\hline 1903 & & & & & & & & 63.5 & & \\
\hline 1904 & & & & & & & & 65.6 & & \\
\hline 1905 & & & & & & & & 68.2 & & \\
\hline 1906 & & & & & & & & 68.0 & & \\
\hline 1907 & & & & & & & & 69.3 & & \\
\hline 1908 & & & & & & & & 70.6 & & \\
\hline 1909 & & & & & & & & 72.6 & & \\
\hline 1910 & & & & & & & & 68.0 & & \\
\hline 1911 & & & & & & & & 66.8 & & \\
\hline 1912 & & & & & & & & 67.6 & & \\
\hline 1913 & & & & & & & & 68.3 & & \\
\hline 1914 & & & & & & & & 70.8 & & \\
\hline 1915 & & & & & 61.9 & & & 73.4 & & \\
\hline 1916 & & & & & 65.4 & & & 74.5 & & \\
\hline 1917 & & & & & 64.7 & & & 78.8 & & 77.0 \\
\hline 1918 & & & & & 70.8 & & & 80.9 & & 74.9 \\
\hline 1919 & & & & & 71.5 & & & 78.7 & & 75.9 \\
\hline 1920 & & & & & 73.2 & & & 77.0 & & 78.5 \\
\hline 1921 & & & & & 72.9 & & & 82.5 & & 84.6 \\
\hline 1922 & & & & & 73.2 & & & 82.7 & & 84.5 \\
\hline 1923 & & & & & 73.2 & & & 81.5 & & 82.4 \\
\hline 1924 & & & & & 73.1 & & & 82.7 & & 81.7 \\
\hline 1925 & & & & & 71.8 & & & 83.8 & & 83.8 \\
\hline 1926 & & & & & 72.1 & & & 83.9 & & 83.3 \\
\hline 1927 & & & & & 74.5 & & & 83.4 & & 84.3 \\
\hline 1928 & & & & & 73.4 & & & 83.0 & & 84.1 \\
\hline 1929 & & & & & 71.0 & & & 82.7 & & 83.4 \\
\hline 1930 & & & & & 74.0 & & & 82.7 & & 86.0 \\
\hline 1931 & & & & & 76.2 & & & 85.7 & & 88.4 \\
\hline 1932 & & & & & 80.8 & & & 87.7 & & 93.3 \\
\hline 1933 & & & & & 81.7 & & & 88.2 & & 94.1 \\
\hline 1934 & & & & & 83.5 & & & 87.8 & & 93.3 \\
\hline 1935 & & & & & 83.0 & & & 87.6 & & 92.7 \\
\hline 1936 & & & & & 82.0 & & & 86.8 & & 92.1 \\
\hline 1937 & & & & & 83.4 & & & 85.5 & & 91.0 \\
\hline 1938 & & & & & 84.4 & & & 85.8 & & 92.5 \\
\hline 1939 & & & & & 82.2 & & & 85.7 & & 92.2 \\
\hline 1940 & & & & & 90.8 & & & 88.9 & & 90.8 \\
\hline 1941 & & & & & 91.3 & & & 90.3 & & 87.1 \\
\hline 1942 & & & & & 92.4 & & & 90.7 & & 82.1 \\
\hline 1943 & & & & & 93.8 & & & 90.4 & & 77.8 \\
\hline
\end{tabular}


Continued from previous page

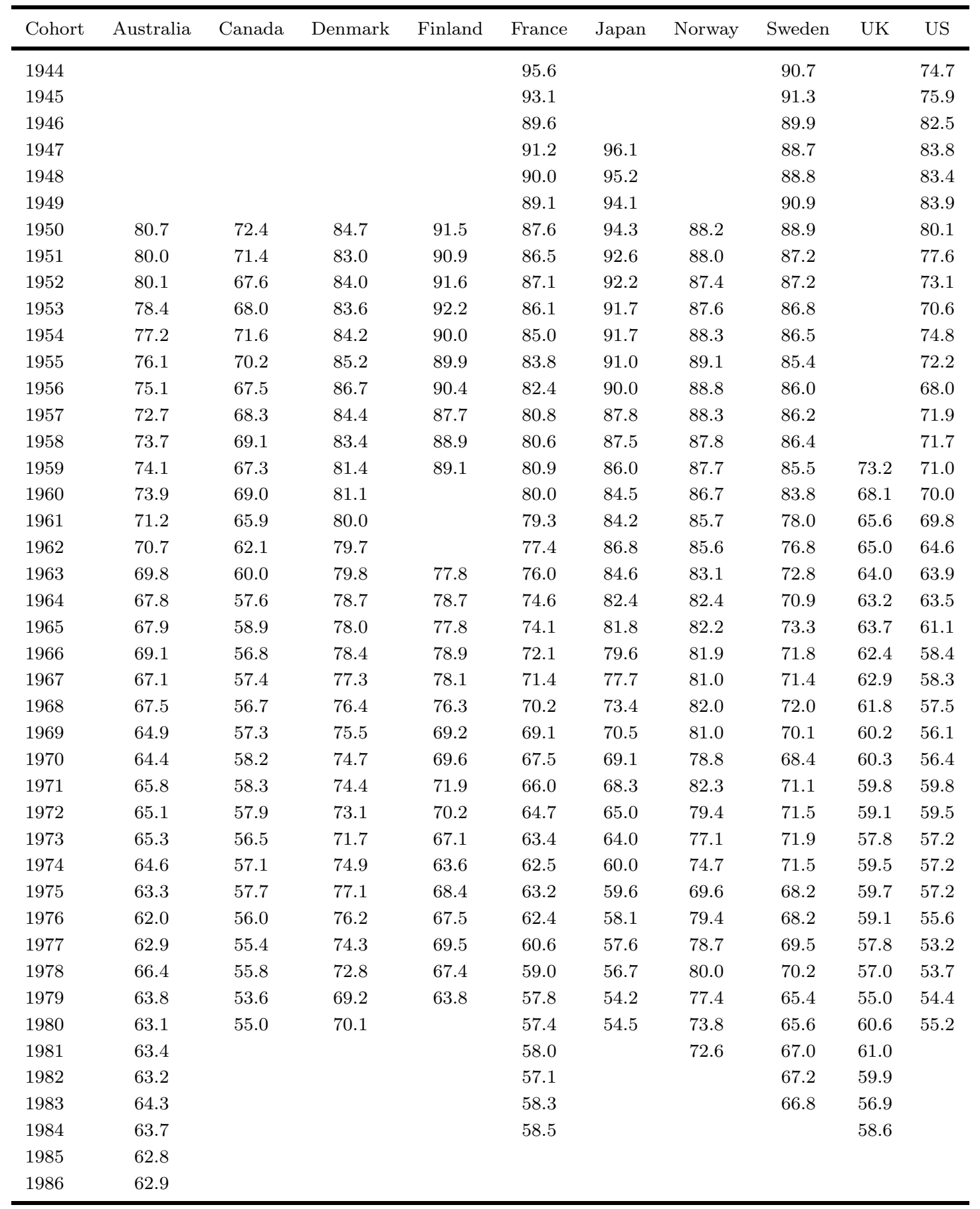


Table 4: Growth and inequality changes contribution to the evolution of absolute mobility from 1940 (or later) onward (see Fig. 3)

\begin{tabular}{lccc}
\hline Country & Decrease in mobility $(p p)$ & Growth contrib. $(p p)$ & Inequality contrib. $(p p)$ \\
\hline Australia & 22.1 & 8.2 & 13.9 \\
Canada & 24.1 & 17.1 & 7.0 \\
Denmark & 17.2 & 14.3 & 2.9 \\
Finland & 30.2 & 18.1 & 12.2 \\
France & 35.5 & 27.9 & 7.7 \\
Japan & 42.2 & 31.1 & 11.1 \\
Norway & 17.7 & 12.7 & 5.1 \\
Sweden & 24.9 & 18.0 & 6.9 \\
UK & 20.0 & 12.5 & 7.5 \\
US & 39.2 & 14.5 & 24.6 \\
\hline
\end{tabular}




\section{Robustness of Absolute Mobility to Changes in Unit of Obser- vation and Income Concept}

Using the detailed data from Garbinti, Goupille-Lebret and Piketty (2018) and Piketty, Saez and Zucman (2018) it is possible to test the robustness of the results for France and the United States to changes in income definition and to different units of observation. In particular, the data from Garbinti, Goupille-Lebret and Piketty (2018) allow comparing absolute mobility for individual adults and equal-split adults in France after 1970. The individualized series assign zero labor income to nonworking spouses. Thus, comparing absolute mobility estimates based on the two different income specifications makes it possible to assess the effect of changes in women's labor force participation and gender inequality on the evolution of absolute mobility. The individualized series is more unequal than the equal-split series by design. Income growth is similar in both. Therefore, the individualized-based estimates should be lower than the baseline estimates. Fig. 10 presents the results, showing that the individualized-based estimates are indeed lower. Yet, the differences between the estimates are small and the average difference is 1 percentage point. This indicates that the rise in female labor force participation rates did not lead to a substantial increase in absolute intergenerational mobility.

The data in Garbinti, Goupille-Lebret and Piketty (2018) also allow considering absolute mobility in France after 1970 for labor income only. The changes in labor income inequality are milder than for total income. This is due to the rising share of capital income in national income after the 1970s (Piketty and Zucman, 2014; Garbinti, Goupille-Lebret and Piketty, 2018). Yet, labor income growth is also slower than for total income. Thus, the differences between the absolute mobility estimates for the two income concepts are expected to be small. This was also found for the United States by Chetty et al. (2017). Detailed labor income data and its distribution are available for the United States from 1962 onward (Piketty, Saez and Zucman, 2018). Fig. 11 presents a comparison between the baseline absolute mobility estimates and labor income-based estimates for France and the United States. We find that absolute mobility for labor income is lower than for total income. However, the differences between the estimates are small and the average difference is less than 1.5 percentage points in both countries. These results, together with those presented in Fig. 10, indicate that the absolute mobility estimates are robust to changes in income definition and to different units of observation (see also Appendix E). This further implies that the detected decrease in absolute mobility is indeed robust. 


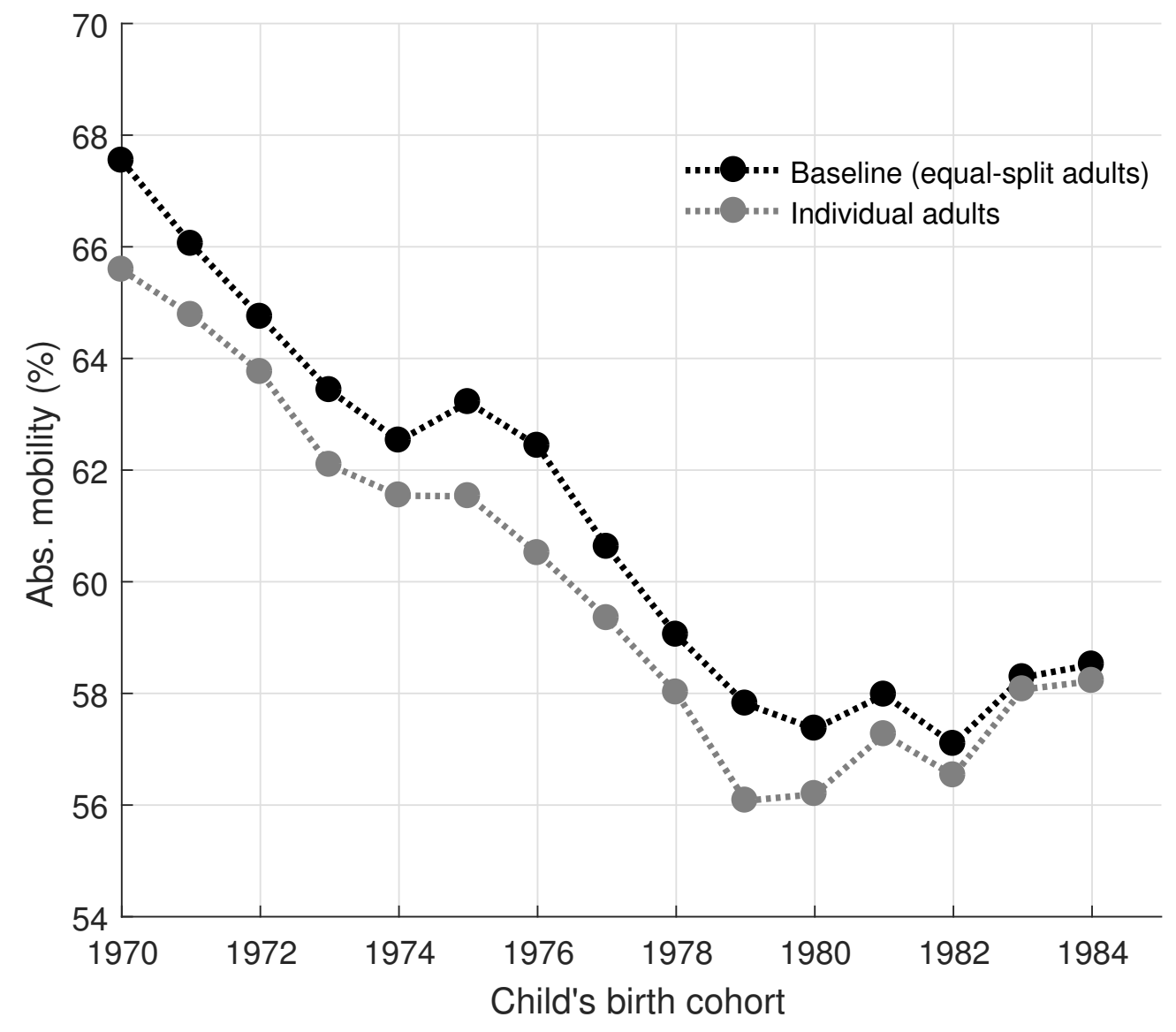

Figure 10: Absolute intergenerational mobility in France using equal-split and individualized income data. 


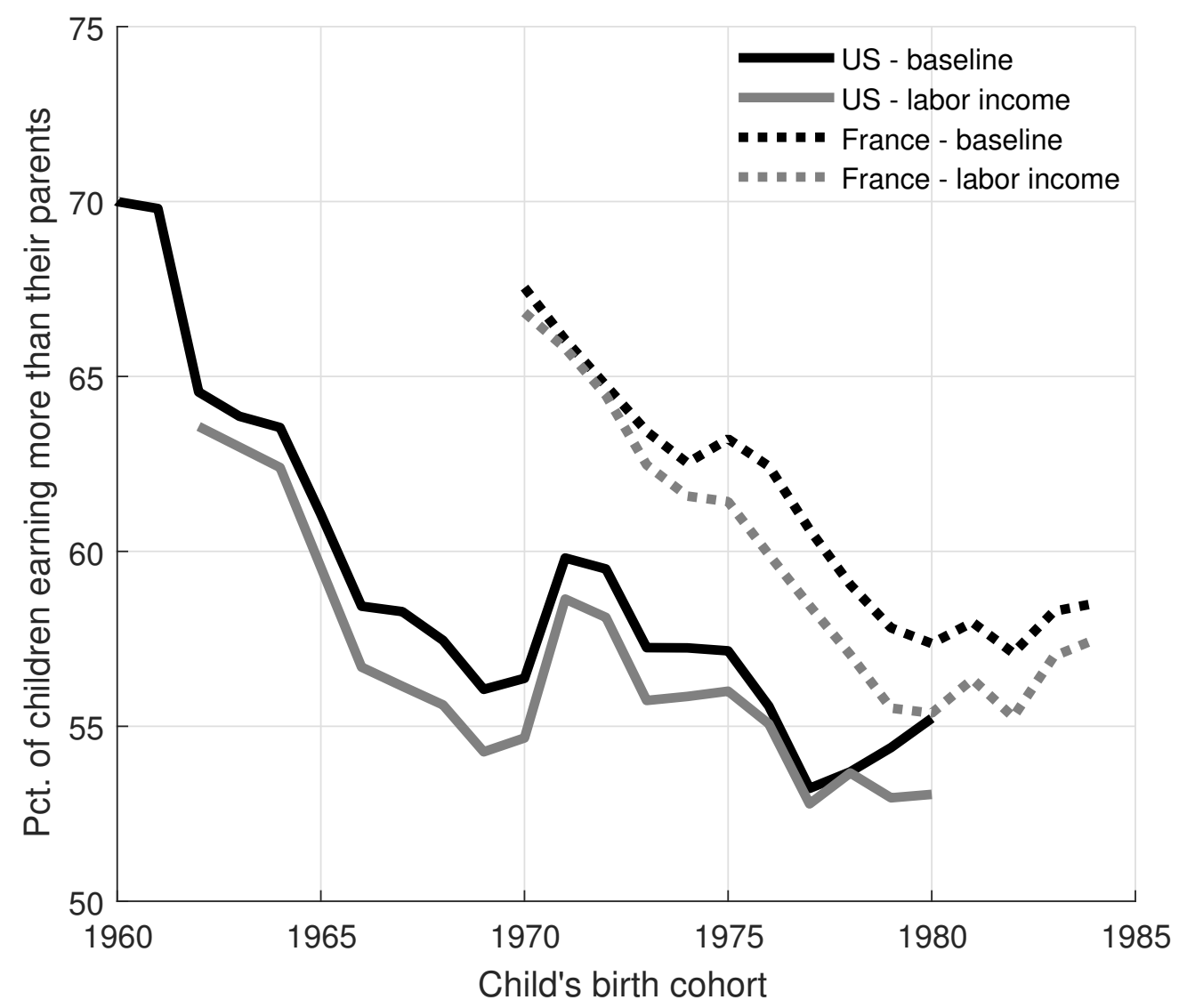

Figure 11: Absolute intergenerational mobility in the United States and in France for total income (black) and labor income (gray). 


\section{J A Comparison between Absolute Mobility and Katz-Krueger Measure of Mobility}

The results of Chetty et al. (2017) show that the share of children earning more than the median parent declined from $92 \%$ in the 1940 birth cohort to $45 \%$ in the 1984 cohort (Katz and Krueger, 2017). This alternative measure of absolute mobility moves almost identically to $A$ across cohorts

in the United States (Katz and Krueger, 2017). Denoting it as $\tilde{A}$, it follows that in the bivariate log-normal model

$$
\tilde{A} \equiv \Phi\left(\frac{\mu_{c}-\mu_{p}}{\sigma_{c}}\right)
$$

where $\Phi$ is the cumulative distribution function of the standard normal distribution.

Using $\tilde{A}$ has obvious advantages over $A$. In particular, they can be "directly computed from standard public-use cross-sectional household survey data and do not require data that longitudinally link children to parents." (Katz and Krueger, 2017) However, $\tilde{A}$ would be close to $A$ only if the IGE is close to $1 / 2$ :

Proposition 2 For a bivariate log-normal distribution with parameters $\mu_{p}, \sigma_{p}$ (for the parents' marginal distribution), $\mu_{c}, \sigma_{c}$ (for the children's marginal distribution) and assuming IGE of $\beta$, then

$$
A=\tilde{A} \Longleftrightarrow \beta=\frac{1}{2}
$$

Proof Following Eq. (A.1)

$$
A=\Phi\left(\frac{\mu_{c}-\mu_{p}}{\sqrt{\sigma_{p}^{2}(1-2 \beta)+\sigma_{c}^{2}}}\right) \text {. }
$$

Following Eq. (J.1)

$$
\tilde{A}=\Phi\left(\frac{\mu_{c}-\mu_{p}}{\sigma_{c}}\right)
$$

and therefore

$$
\tilde{A}=A \Longleftrightarrow \frac{\mu_{c}-\mu_{p}}{\sqrt{\sigma_{p}^{2}(1-2 \beta)+\sigma_{c}^{2}}}= \pm \frac{\mu_{c}-\mu_{p}}{\sigma_{c}}
$$

We then obtain 


$$
\frac{\mu_{c}-\mu_{p}}{\sqrt{\sigma_{p}^{2}(1-2 \beta)+\sigma_{c}^{2}}}= \pm \frac{\mu_{c}-\mu_{p}}{\sigma_{c}} \Longleftrightarrow \sigma_{c}= \pm \sqrt{\sigma_{p}^{2}(1-2 \beta)+\sigma_{c}^{2}} \Longleftrightarrow \beta=\frac{1}{2}
$$

It is therefore no surprise that for the United States $A$ and $\tilde{A}$ are similar - Aaronson and Mazumder (2008) estimate the IGE for the 1950-1970 birth cohorts at 0.46-0.58. In countries such as Canada or Denmark, in which the IGE is substantially lower than 0.5 (Corak, 2013), using $\tilde{A}$ neglects the part high relative mobility plays in determining $A$. This will lead to overestimation of absolute mobility if measured this way. For example, in Denmark, the average estimated $A$ for 1950-1980 birth cohorts is $78.5 \%$. If $\tilde{A}$ is considered, the average value is $87.2 \%$. For the United States this difference would be considerably smaller: $62.2 \%$ and $63.7 \%$, respectively. Setting aside the normative question of which measure of absolute intergenerational mobility is of most interest, we emphasize that $\tilde{A}$ cannot be used as a proxy for $A$, unless the IGE is close to 0.5 . 